\title{
Neural coding and contextual influences in the whisker system
}

Rasmus S. Petersen ${ }^{1 *}$, Stefano Panzeri ${ }^{12}$, Miguel Maravall ${ }^{3}$

${ }^{1}$ Faculty of Life Sciences, University of Manchester, UK

${ }^{2}$ Italian Institute of Technology, Robotics, Brain and Cognitive Sciences Department, Via Morego, 30., 16163 Genova, Italy

${ }^{3}$ Instituto de Neurociencias de Alicante UMH-CSIC, Sant Joan d'Alacant, Spain

* Corresponding author

r.petersen@manchester.ac.uk

telephone +441613065921 


\section{Abstract}

A fundamental problem in neuroscience, to which Segundo has made seminal contributions, is to understand how action potentials represent events in the external world. The aim of this paper is to review the issue of neural coding in the context of the rodent whiskers, an increasingly popular model system. Key issues we consider are: the role of spike timing; mechanisms of spike timing; decoding and context-dependence. Significant insight has come from the development of rigorous, information theoretic frameworks for tackling these questions, in conjunction with suitably designed experiments. We review both the theory and experimental studies. In contrast to the classical view that neurons are noisy and unreliable, it is becoming clear that many neurons in the whisker pathway are remarkably reliable and, by virtue of spike timing with millisecond-precision, have high bandwidth for conveying sensory information. In this way, even small ( 200 neuron) subcortical modules are able to support the sensory processing underlying sophisticated whisker-dependent behaviours. Future work on neural coding in cortex will need to consider new findings that responses are highly dependent on context, including behavioural and internal states.

\section{Acknowledgements}

We thank E. Arabzadeh, M.E. Diamond, M.A. Montemurro for stimulating collaboration. For financial support, we thank: HFSP RG0043/2004, Spanish Ministry of Education and Science BFU2006-04791/BFI (co-funded by the European Regional Development Fund), CONSOLIDER CSD2007-00023, IIT, EPSRC EP/C010841, EP/E002331 and EP/E057101. 


\section{Introduction}

Understanding how action potentials (“spikes”) represent events in the external world is a fundamental aim of neuroscience. Since spike trains evoked by the same stimulus tend to be variable, the relation between a stimulus $\mathbf{s}$ and response $\mathbf{r}$ is given by the "probabilistic dictionary” $\mathrm{P}(\mathbf{r} \mid \mathbf{s})$ (Rieke et al. 1997). One key aim of neural coding research is to understand the essential structure of this dictionary. However, since both stimuli and responses tend to be complex, it is far from obvious how to find it. Traditionally, researchers have used simple descriptors of $\mathbf{r}$ such as the number of spikes fired within a given time window ("spike count”). Adrian, studying muscle stretch receptors in the frog, first showed that the spike count revealed important dictionary structure (Adrian 1926), and counting spikes recorded from single neurons has been a productive research strategy ever since.

However, researchers have been aware for a long time that more complex and more powerful codes are possible. Segundo and colleagues were amongst the first to recognize this. As stated in their 1966 review:

“...there is an enormous wealth of information about the structure and function of the nervous system which can be derived from careful study of the detailed timings of spike events” (Moore et al. 1966).

Indeed, as discussed below, Segundo carried out seminal work to show that coding by spike timing was not only a theoretical possibility (MacKay and McCulloch 1952), but an experimental reality (Bryant and Segundo 1976). Given Segundo’s important contributions to the area of neural coding, it is a privilege to contribute a paper on the topic to this special issue in his honour.

\section{Coding by spike count vs coding by spike timing}

Is spike timing reliable enough to underlie a robust neural code? Microelectrode recording experiments on the visual cortex in the 70s and 80s established that the response of a neuron to repeated presentation of a drifting grating typically exhibits Geiger-counter type variability across trials (variance of the spike count approximately equal to its mean) (e.g., Tolhurst et al. 1983). This suggested that neurons are too noisy for an event such as the occurrence of a single spike at a precise time to play a significant role in coding. The view thus emerged that neurons are noisy elements only capable of supporting a low capacity, yet robust, spike count code (Shadlen and Newsome 1994). 
Other studies challenged this view. In one relevant type of experiment, current is injected directly into a neuron through an intracellular microelectrode and the neuron's spiking response recorded. In a standard protocol, the stimulus is a step of depolarizing current which, post-onset, is constant in time. Consistent with what one might expect from the noisy neuron view, this stimulus evokes responses in which spike times vary considerably from trial to trial (e.g., Mainen and Sejnowski 1995). In contrast, if the injected current fluctuates rapidly (e.g., white noise), the evoked spike trains can be highly reproducible. This was first shown by Segundo in aplysia neurons (Bryant and Segundo 1976) and later shown also to apply to neocortical neurons in vitro (Mainen and Sejnowski 1995). Related results have been obtained from in vivo work on the fly visual system (de Ruyter van Steveninck et al. 1997). The fly has two H1 neurons which detect horizontal, wide field motion (one for leftwards motion, the other for rightwards motion). Analogously to injection of constant current, if a bar of light is moved at constant velocity across the fly's visual field, H1 responds vigorously, but with spikes whose precise times vary from trial to trial. In contrast, if the velocity continuously changes (white noise), the evoked spike trains are highly reproducible.

These findings have two profound implications. First, they demonstrate that the fundamental biophysical machinery of neurons is able to support coding by precise spike times - noisy spike trains are not inevitable. Second, they demonstrate that the same neuron can respond noisily or not, depending on the stimulus conditions. Which stimuli, then, should be used in studies of neural coding? A useful strategy is to be guided by an animal's natural behaviour. In the fly, natural flight paths consist of frequent and rapid turns (Land and Collett 1974). Long periods of constant velocity stimulation are rare, and the fluctuating velocity case, modeled by white noise, is more realistic. This suggests that, at least for fly H1, coding by precise spike timing in a low noise regime is the biologically relevant case.

In a spike timing code, the number of messages that can be transmitted increases exponentially with spike timing precision (MacKay and McCulloch 1952). Such high capacity means that small numbers of neurons can transmit enough information to underpin complex sensory-guided behaviours, such as object identification. Moreover, since the information per spike is high, such codes can also be energetically efficient. Since neural activity accounts for a significant fraction of the body's energy budget this may be an important evolutionary consideration (Attwell and Laughlin 2001). 
In sum, the debate on spike count vs spike timing codes is important, since the two hypotheses imply very different views of how neurons compute and how neural circuits function. From the perspective of spike count coding, neurons are unreliable, low bandwidth computing elements, and large numbers are consequently necessary for robust information processing. From the perspective of H1-type spike time coding, neurons are reliable, high bandwidth elements, and demanding information processing tasks can be performed by small neural circuits.

The invertebrate data force us to consider seriously the possibility that the 'noisy neuron' view of brain function may be incorrect. However, it might of course be that invertebrate brains work differently to mammalian ones. An important challenge is to find out what principles of neural coding apply to the mammalian brain under natural conditions. To make progress, we need good model systems. Since the seminal discovery of "barrels" in rodent primary somatosensory cortex (Woolsey and Van der Loos 1970), an increasingly useful and popular model system is the whisker system. A number of excellent reviews have recently been published on whisker neurobiology (Alloway 2008; Brecht 2007; Diamond et al. 2008b; Petersen 2007). The aim of this paper is to complement these reviews by focussing specifically on what is known about sensory coding in the whisker system. Much of what is quantitatively known about the nature of the neural code has been measured under anaesthetized conditions, where both whisker position and brain state are under precise experimental control. In this review, we first focus on the data available for the most precisely controlled conditions, analysed using the information theoretic approach. However, contextual influences, ranging from the sensory environment to the animal's state of attention, are likely to alter the nature of the neural code. In the final part of the review, we therefore discuss contextual effects, including cases where the effects on information coding have not been explicitly measured but are likely to be important. We start by providing a brief overview of whisker neurobiology.

\section{The rodent whisker system}

\section{Whiskers and whisking behaviour}

Rodents are nocturnal animals which tend to inhabit confined spaces underground. To sense the nature of this environment, they have evolved a sensitive array of 30 or so whiskers on each side of the snout. The whiskers are arranged in a highly consistent, grid-like pattern. Their locations are specified by 'row' and 'arc' coordinates, where the rows are labeled by the 
letters A-E and arcs by numbers (Fig. 1A). When a rodent is exploring an object of interest, it sweeps its whiskers back and forth across the surface at around $10 \mathrm{~Hz}$ - a behaviour known as whisking. In this way, animals can make extremely fine discriminations between alternative objects. For example, blind-folded rats have been trained to discriminate between two objects which differ only in the fact that one has a smooth surface, whereas the other is machined with a spiral groove $50 \mu \mathrm{m}$ deep (Carvell and Simons 1990). In fact, rats can be trained to perform this task using only a single whisker (Carvell and Simons 1995). By human standards, this task is demanding, and rat whisker discrimination performance is comparable to that of humans using their finger tips.

\section{FIGURE 1 AROUND HERE}

When rats whisk the surface of an object, the whisker-object contact induces a complex whisker vibration. This vibration is influenced both by the animal's active control of whisking, the frequency of which varies in the range 5-25 Hz (see Kleinfeld et al. 2006), by the structure of the object surface and by the mechanics of the whiskers themselves. Object surface structure is a principal influence on whisker vibration. For example, whisking sandpaper of a given roughness elicits a pattern of damped whisker vibration ("kinetic signature”) that is both reproducible and characteristic of the surface (Arabzadeh et al. 2005). How kinetic signatures are transduced by whisker mechanoreceptors is likely to depend on whisker mechanics (Andermann et al. 2004; Ritt et al. 2008). In the typical laboratory rat, whiskers are 10-60 mm long and taper from 100-200 $\mu \mathrm{m}$ diameter at the base to 5-40 $\mu \mathrm{m}$ at the tip (Neimark et al. 2003). Whiskers transmit energy to the follicle where the mechanoreceptors are located, in a manner that depends on their mechanical properties: for example, they have resonant modes that are length-dependent and range from $100 \mathrm{~Hz}$ for the long caudal whiskers to $600 \mathrm{~Hz}$ for the short rostral ones (Hartmann et al. 2003; Neimark et al. 2003). The precise significance of whisker resonance is under debate (Diamond et al. 2008a; Ritt et al. 2008). The emerging picture is that whisking an object may "encode” its surface structure as a corresponding pattern of whisker vibration. Consistent with this view, the different grades of sandpaper studied by Arabzadeh et al. can be statistically discriminated purely from optical measurements of the induced whisker vibrations in the frequency band 30-150 Hz (Hipp et al. 2006).

Transduction of information-bearing whisker vibrations into neural signals occurs in mechanoreceptors, located in the whisker follicle (see Rice and Munger 1986). These mechanoreceptors are notable for an almost complete absence of spontaneous activity (Gibson 
and Welker 1983; Zucker and Welker 1969). Although they vary considerably in activation threshold, many mechanoreceptors are remarkably sensitive. When stimulated with white noise whisker vibration, their spike trains are highly reproducible (Jones et al. 2004b), the spikes timed to sub-millisecond precision (Fig. 2). Even without quantitative analysis, it is clear that such neurons have a high bandwidth for transmitting information. In this respect they resemble fly $\mathrm{H} 1$ more than they do mammalian visual cortical neurons.

\section{FIGURE 2 AROUND HERE}

\section{Neuroanatomy of the whisker system}

Whisker mechanoreceptors are innervated by a branch of the trigeminal nerve, and their cell bodies are located in the trigeminal ganglion. The ganglion is partially topographically organized. Cell bodies of neurons innervating A row whiskers are located dorsally, those innervating E row whiskers ventrally. However, there is no apparent topographic order for the arcs (Leiser and Moxon 2006). This is in marked contrast to higher stations of the whisker pathway. Ganglion neurons project in parallel to a cluster of nuclei, known as the brainstem trigeminal complex. Recent research has shown that there are parallel pathways that convey whisker information from the brainstem to the cortex: for details, see (Alloway 2008; Bezdudnaya and Keller 2008; Pierret et al. 2000; Urbain and Deschenes 2007). In outline, neurons in some brainstem nuclei are clustered into 'barrelettes' (Belford and Killackey 1979) and these nuclei contain precise topographic maps, where each of the barrelettes is in one-to-one correspondence with a particular whisker. Barrelettes project to corresponding 'barreloids' of the ventro-posterior medial nucleus of the thalamus (VPM) (van der Loos 1976), which project in turn to the 'barrels' (Woolsey and Van der Loos 1970) of layer IV of primary somatosensory cortex (or "barrel cortex"). Thus, at each level of the system, there are one or more anatomical maps, in one-to-one correspondence with the arrangement of the whiskers on the snout (Fig. 1). However, there is some convergence in the projections so, although neurons within a given barrel, barreloid or barrelette spike best to deflection of the so-called 'principal whisker', they respond also, albeit to a lesser degree, to deflection of one or more 'surround whiskers'. Indeed, at the subthreshold level, receptive fields can be large, encompassing most of the whisker pad (Brecht and Sakmann 2002; Moore and Nelson 1998). In contrast, to the precise topography of the barrelette-barreloid-barrel pathways, a separate 'paralemniscal' pathway through the posterior nucleus of the thalamus (POM) is less spatially specific (see Alloway 2008). 
The architecture of the whisker pathway is similar to that of other sensory modalities (excepting olfaction) in that it includes a thalamic stage linking the receptor and cortical levels, and involves an expansion in the number of neurons from each level to the next. In mouse, there are around 100 large myelinated fibres per whisker (Lee and Woolsey 1975; Welker and Van der Loos 1986), in rat around 30\% more (Rice et al. 1986). The ratio of neurons per whisker in the thalamus is of the same order of magnitude: the estimate from rat is 250 neurons per VPM barreloid (Land and Simons 1985). In contrast, from VPM to barrel cortex, there is a dramatic expansion. Considering just layer IV, which contains 2000 neurons per barrel in mouse, there are 20 times more neurons per whisker than in the periphery. Considering the whole barrel-column (a cylinder with the same cross-sectional area as a barrel, spanning all cortical layers), the ratio is 60 times (Welker and Van der Loos 1986). These trends may be compared with the cat/primate visual system (reviewed by Peters and Payne 1993). In cat, there are 4 neurons per retinal ganglion cell in LGN, 100 neurons per retinal ganglion cell in layer IV of primary visual cortex. In monkey, the numbers are 1 and 56 respectively. The functions subserved by this expansion are not fully understood either in the whisker pathway or in other modalities. However, since subcortical stations reliably represent basic stimulus features such as location on the whisker pad, it is likely that the role of expansion is to help the cortex fulfill more sophisticated aspects of sensory processing, such as coding higher order stimuli (e.g., collective whisker motion) or adapting the code to current behavioural needs.

\section{Sensory features represented in the whisker system}

To probe what stimulus parameters are represented in the whisker system, many studies have used a "ramp-and-hold" stimulus, where a whisker is deflected in a given direction at a desired slope until it reaches some steady-state amplitude. The steady-state is maintained for a certain period before the whisker is returned to its initial position. Using ramp-and-hold stimuli, it has been shown that neurons in the whisker system are sensitive to the location of a stimulus on the whisker pad, the direction of deflection, the slope of deflection and the repetition rate (Simons 1978; Zucker and Welker 1969). Since the slope of a ramp is proportional to deflection velocity, one interpretation of these data is that neurons encode velocity. Recent studies of barrel cortex using sinusoidal and texture stimuli also indicate velocity coding (Arabzadeh et al. 2003; Arabzadeh et al. 2005). Note, however, that, in a ramp-and-hold stimulus, ramp velocity covaries with acceleration (and all higher derivatives of whisker position) at ramp onset. These parameters can be decoupled by a white noise, 
reverse correlation approach but this topic is outside the scope of this review: see (Petersen et al. 2008).

Many of the above studies measured neuronal responses in terms of the spike count (typically measured over $100 \mathrm{~ms}$ or so, and averaged over stimulus repetitions). The fact that spike count is modulated by a number of important stimulus parameters makes it a plausible candidate for a neural code. However, unlike experimenters, animals do not do trialaveraging when they are making behavioural decisions. For example, rats take only 1-3 whisks to discriminate a rough surface from a smooth one (von Heimendahl et al. 2007). Animals essentially make decisions on the basis of single trial responses. This means that an effective neural code must be robust to random, trial-to-trial response fluctuations. Consequently, data on tuning curves do not speak to the issues of central concern here. To make progress, it is essential to compare alternative codes using a metric that quantifies their ability to discriminate alternative stimuli on a single trial basis.

\section{Information theoretic framework for sensory coding}

"The promise held out to students of the nervous system by information theory (statistical communication theory) since the publication nearly two decades ago of the classic monograph of Shannon \& Weaver has been realized to a disappointingly small extent. Nevertheless, the framework of that theory still seems a natural one to describe those aspects of neuronal function which are concerned with communication and information processing” (Moore et al. 1966)

Although, as noted above, tuning curves (trial-averaged spike count as a function of some stimulus parameter) are an important tool, their limitation from the point of view of neural coding is that they do not take into account the effect of response variability on information transmission. The simplest metric that remedies this $\left(d^{\prime}\right)$ is the difference in mean spike count evoked by two stimuli, divided by the standard deviation of the spike count. $d^{\prime}$ can readily be generalised to multiple bins/neurons. It is a simple and useful approach (for an application to the whisker system, see Petersen and Diamond 2000). However, $d^{\prime}$ assumes that response variability is Gaussian distributed. It can be desirable to avoid this assumption and adopt a more general approach. 


\section{Mutual information}

Mutual information measures the maximal reduction of uncertainty about which stimulus $\mathbf{s}$ occurred that can be gained by observing the neural response $\mathbf{r}$ on a single trial. It, is defined as follows (see Cover and Thomas 1991; Rieke et al. 1997):

$$
I(S ; R)=\sum_{\mathbf{r}, \mathbf{s}} \mathrm{P}(\mathbf{r}, \mathbf{s}) \log _{2} \frac{\mathrm{P}(\mathbf{r}, \mathbf{s})}{\mathrm{P}(\mathbf{r}) \mathrm{P}(\mathbf{s})}
$$

Here $S$ is the set of stimuli $\{\mathbf{s}\}$ and $R$ is the set of responses $\{\mathbf{r}\}$. Mutual information is measured in units of bits: 1 bit corresponds to a reduction of uncertainty by a factor of 2 . The main advantage of $I(S ; R)$ is that it includes the effect of all possible moments of the stimulusresponse probability that might be relevant, not only the mean response and the variance. An important caveat is that downstream neural systems may not necessarily be able to access all information present in a neural response. We will give examples later of how this 'decoding' issue has been addressed, both theoretically and experimentally.

An important conceptual property of mutual information is that it does not presuppose any specific assumptions about which stimulus parameters a given neuron encodes. This enables us to separate the general task of neural coding into a distinct (1) "response question” and (2) "stimulus question". These are: (1) what are the key parameters of $\mathbf{r}$ (information-bearing elements) that encode information? (2) What stimulus parameters do these informationbearing elements encode? Here, we concentrate primarily on the response question.

\section{Practical application of information theory to spike train data}

To apply information theory to spike data, it is first necessary to quantify the response $\mathbf{r}$. In the simplest case, an experiment consists of a discrete set of stimuli (e.g., deflection of whisker D1 vs that of D2), each of which is presented on multiple trials. Defining stimulus onset as time 0 , the response is typically measured within a time window $(0, T)$. In the case of a single neuron spike count code, the response is the number of spikes fired within the time window (Fig. 3B,E). These data are then used to make histogram estimates of $\mathrm{P}(\mathbf{r} \mid \mathbf{s})$. We know $\mathrm{P}(\mathbf{s})$ from the design of the experiment, and can derive $\mathrm{P}(\mathbf{r})$ from the identity $\mathrm{P}(\mathbf{r})=\sum_{\mathbf{r}} \mathrm{P}(\mathbf{r} \mid \mathbf{s}) \mathrm{P}(\mathbf{s})$. At first sight, estimating the mutual information conveyed by the spike count seems then a simple matter of plugging these probability estimates into equation 1. 
The procedure for the spike timing code is similar. To quantify the spike timing response, the $(0, T)$ window is subdivided into bins of width $\Delta t$ and the number of spikes occurring in each bin is registered (Fig. 3C,F). Thus, each possible response $\mathbf{r}$ is represented by a "word" of length $L=T / \Delta t$. If $\Delta t$ is less than or equal to the refractory period, each of the bins will contain 0 spikes or 1 spike, and the words will be binary. As before, the probability of each possible word conditional on each stimulus can be estimated, yielding $\mathrm{P}(\mathbf{r} \mid \mathbf{s})$ and $\mathrm{P}(\mathbf{r})$, which can again be plugged into equation 1 .

\section{FIGURE 3 AROUND HERE}

The simplicity of this procedure is deceptive. Applying information theory to real experimental data is non-trivial. Due to practical constraints, the ratio of trials available to parameters that must be estimated tends to be small. The conditional probabilities therefore tend to be subject to considerable sampling error. Sampling error translates into positive bias of the mutual information estimate, known as 'sampling bias'. The danger comes from the fact that, the more parameters there are to estimate, the worse is the bias. When comparing a complex code (many parameters) to a simple code (few parameters), the mutual information estimate for the complex code may be larger simply due to sampling bias. For many years, this sampling bias problem was a significant obstacle and was one reason why the promise of information theory was, as Segundo and colleagues put it in the quotation above, "realized to a disappointingly small extent”. Recently, however, there has been substantial progress: both in the theory of sampling bias and, consequently, in the development of practical bias correction procedures (reviewed by Panzeri et al. 2007). Provided care is taken to use these algorithms within their domain of applicability, it is possible to obtain accurate mutual information estimates from one or more neurons. In the next section, we review work applying the information theoretic strategy to address the question of whether neurons in the whisker system encode sensory information by spike count or by spike timing.

\section{Coding by spike count vs spike timing in the whisker system}

\section{Role of the first spike}

A remarkable feature of the whisker system is that processing is very quick. A response to whisker deflection is detectable in barrel cortex with a latency of $5 \mathrm{~ms}$. However, the total duration of the cortical response is $30-60$ ms (Petersen and Diamond 2000) and long time 
windows are consequently often used to measure the response. Given the survival value of rapid information processing, an interesting question is how soon the response becomes useful - that is, how soon can it support reliable stimulus discrimination on a single trial basis. Petersen and Diamond (2000) recorded multi-unit responses to whisker deflection using a 100 microelectrode array implanted in barrel cortex. They used the population $d^{\prime}$ measure to assess how well the spike count measured in a given time window supported discrimination between deflection of different whiskers. The result was that $d^{\prime}$ typically peaks within $16 \mathrm{~ms}$ of stimulus onset. These data suggested that the early part of the cortical response is crucial.

To investigate the neural code for stimulus location in more detail, subsequent studies applied an information theoretic approach to single unit data (for review see Panzeri et al. 2003; Petersen and Panzeri 2003). Panzeri et al. (2001) and Petersen et al. (2001) quantified the response to whisker deflection in terms of spike count or spike timing, as described above. An important aspect of these data (consistent with other studies) was that the average spike count was low enough ( 0.3 spikes per $40 \mathrm{~ms}$ window) to accurately approximate the mutual information by a second order power series expansion in time (Panzeri and Schultz 2001). In this "series expansion method", the mutual information estimate depends only on the first and second moments of $\mathrm{P}(\mathbf{r} \mid \mathbf{s})$. This substantially reduces the number of parameters that must be estimated, so that the series expansion has much better sampling properties than direct application of Equation 1. This makes it possible to estimate spike timing information at higher temporal resolution than would otherwise be possible.

In this way, it was found that single neurons convey on average $44 \%$ more information by spike timing than by spike count (response window $40 \mathrm{~ms}$, bin size $5 \mathrm{~ms}$ ) (Panzeri et al. 2001). To pin down where this spike timing information comes from, the analysis was repeated using only the first spike fired on each trial, only the second spike or only the third spike. Most of the spike train information could be attributed to the first spike per trial. For example, the first spike conveys $91 \%$ of the spike train information, the second spike $43 \%$, the third spike 18\% (Petersen et al. 2001). The fact that these percentages sum to more than $100 \%$ shows that the later spikes are redundant with the first. Moreover, it is the timing of the first spike, not merely its presence/absence, that is crucial, since the former conveys $210 \%$ more information than the latter (Panzeri et al. 2001). These conclusions are robust to precise details of the time window, bin size, neuronal location and stimulus set employed (Petersen et al. 2002). This suggests that this spike timing code is a simple one: the key coding element is the time of the first post-stimulus spike. Thus, consistent with the results of Petersen and Diamond (2000), the early part of the cortical response is the most informative. This principle 
applies not only to the whisker system but to many sensory systems (Foffani et al. 2004; Heil 2004; Johansson and Birznieks 2004).

\section{Coding of time-dependent stimuli}

Studies such as those considered in the previous section probe the response of the system to transient events. In contrast, texture coding involves temporally extended whisker vibrations, for which different principles of coding might apply. Arabzadeh el al. (2004; 2003) recorded the response of multi-unit clusters in barrel cortex to sinusoidal vibrations of varying frequency (19-341 Hz) and amplitude. They computed the information that spike count conveyed about these stimulus parameters. By systematically varying the response window, they found that information is detectable by 6 ms post stimulus onset and reaches a peak 2030 ms post stimulus onset. This suggests that the principle of rapid information processing in this system applies not just to transient whisker deflection stimuli but also to temporally extended whisker vibrations.

What is the precise nature of the neural code for temporally extended stimuli? In principle, direct application of Equation 1 could be used to study texture coding. The problem is that the set of possible textures is not a small discrete set (as with the whisker location problem) but a very large one. To get a feeling for this, we can make a rough calculation. Suppose that a texture is represented by vibration over one whisking cycle (ca $100 \mathrm{~ms}$ ) and that frequencies up to $150 \mathrm{~Hz}$ are relevant (Hipp et al. 2006). To preserve this information, the minimum (Nyquist) sampling frequency is $300 \mathrm{~Hz}$, which implies 30 samples per whisking cycle. Thus, the space of possible vibrations for one whisker is perhaps 30 dimensional. (If other whiskers convey additional information, it could be considerably larger). This space is far too large to sample by systematic search: the dictionary $\mathrm{P}(\mathbf{r} \mid \mathbf{s})$ would consist of so many parameters as to render the information theoretic sampling problem hopeless.

To make progress, researchers have used three complementary approaches. The first is to pick a small discrete set of textures and consider the problem of how differences between the chosen textures are encoded, on the assumption that this set is representative. In this case the theoretical problem reduces to the tractable discrete case already considered. The second is an ergodic sampling procedure. The third is a stimulus reconstruction procedure. We discuss each approach in turn.

Arabzadeh et al. (Arabzadeh et al. 2006) measured the response of single neurons in the trigeminal ganglion and multi-unit clusters in barrel cortex to the whisker vibrations induced 
by the whiskers sweeping either through the air, across a smooth object (a CD) or across four sandpapers of varying roughness. They measured the mutual information between the spike count and a stimulus set consisting of a given pair of stimuli. In cortex, they found mutual information to be 0.6 bits when the stimulus set consisted of a sandpaper and CD but near zero when it consisted of two sandpapers of distinct but similar roughness. Consistent with the previous findings on rapid processing discussed above, the spike count information (averaged over stimulus pairs) increases rapidly at the beginning of the whisking cycle, reaching a peak in cortex only 16 ms later.

These data suggest that a simple spike count code is viable for a coarse discrimination such as that between a rough and a smooth surface. However, behaviourally, rats can reliably discriminate between different rough surfaces (Carvell and Simons 1990; Guic-Robles et al. 1989). Arabzadeh et al. (2006) noted that the sandpapers that elicited similar average spike counts, did evoke differences in PSTHs. This suggests a spike timing code. To test whether the PSTH differences were robust to trial-to-trial response variability, they measured the mutual information that spike timing (24 ms time window, $4 \mathrm{~ms}$ bins) conveyed about stimulus pairs. For rough vs smooth cases, spike timing adds little information to that available in the spike count. However, for the rough vs rough cases, spike timing adds $110 \%$ 150\% mutual information (barrel cortex, ganglion respectively), a major increase both for ganglion and cortex. These data indicate that spike timing might be extremely important for making discriminations between certain classes of texture. The estimated advantage of spike timing is likely to be conservative, at least for the ganglion, for two reasons. First, in ganglion, even with $4 \mathrm{~ms}$ bins, spike timing information frequently saturated the stimulus entropy limit of 1 bit. Second, there is evidence that spike timing precision in ganglion can be as low as $0.1 \mathrm{~ms}$ (Fig. 2, Arabzadeh et al. 2005) - i.e., 40 times higher than the $4 \mathrm{~ms}$ bin size. Thus, with a larger stimulus set, probed at sub-millisecond precision, it is likely that the spike timing information advantage would be considerably greater.

A second way (de Ruyter van Steveninck et al. 1997; Strong et al. 1998) to consider coding of time-dependent stimuli is as follows. Instead of presenting a small set of short temporal stimuli in turn, many such stimuli can be strung together into one long stimulus. A convenient way to do this is to generate a sequence of Gaussian white noise. Since white noise is uncorrelated in time, a long sequence will explore the space of possible temporal patterns in an efficient and unbiased way. This means that, during the course of the white noise sequence, each time point $t$ can be considered to mark the start of a different stimulus $\mathbf{s}$. Using the procedure of Fig. 3, the response $\mathbf{r}$ of duration $T$ (spike count or spike timing) can 
be measured starting at each time $t$. By recording the neuronal response to many repetitions of the white noise stimulus, the data can be used to estimate the probability $\mathrm{P}(\mathbf{r} \mid t)$ at each time $t$. Through the s- $t$ correspondence, $\mathrm{P}(\mathbf{r} \mid t)$ can be thought of as a stimulus-conditional response probability. Therefore, the mutual information can be estimated using the formula:

$I(S ; R)=\left\langle\sum_{\mathbf{r}} \mathrm{P}(\mathbf{r} \mid t) \log _{2} \frac{\mathrm{P}(\mathbf{r} \mid t)}{\mathrm{P}(\mathbf{r})}\right\rangle_{t}$

where the angled brackets denote an average over $t$. The essence of this method, shown by comparison with Equation 1, is that the multi-dimensional integral over $\mathrm{P}(\mathbf{s})$ has been replaced by an integral over time. The method assumes that the time-scale of neuronal stimulus integration is short with respect to the trial duration.

To study VPM thalamus, Montemurro et al. (2007a) used this approach, in conjunction with a new procedure for correcting sampling bias that permitted increased temporal precision (Montemurro et al. 2007b). They reported first that VPM neurons respond in a reliable way to white noise whisker deflection. The average jitter of the spike times from trial to trial was $0.4 \mathrm{~ms}$, indicating the potential for a high bandwidth code. To find out whether spike timing is robust enough to carry information on single trials, the authors estimated the mutual information between stimulus and response. They found that, on average, neurons convey 9.3 times more information by spike timing (60 ms response window, $5 \mathrm{~ms}$ bins) compared to spike count. Information increases with bin resolution up to at least $0.5 \mathrm{~ms}$ (maximum across neurons 77.9 bits/s, average 26.5 bits/s).

To interpret these numbers, it is useful to make a rough calculation. Consider a rat trying to identify some object from a set of many alternatives. As noted above, rats can often acquire the sensory information they need to solve a discrimination tasks in a single whisk. Suppose, therefore, that the relevant response window is $100 \mathrm{~ms}$. In this period, the best recorded VPM neuron conveys 7.8 bits, an average one 2.7 bits. This amount of information narrows down the uncertainty about the object by $2^{7.8}$ and $2^{2.7}$ times respectively. Thus, supposing the rat can discriminate between $10^{6}=2^{19.6}$ equally likely objects, this could be done on the basis of as few as three best neurons or eight average ones, assuming that those neurons convey independent information and that all information they convey is decoded by neural circuits downstream. For discussion of decoding, see below.

Thus, under white noise stimulus conditions, precise spike timing permits neurons in the subcortical whisker pathway to convey a large amount of information. In principle, a given amount of information might be spread thinly over many spikes (a diffuse code) or 
concentrated in few spikes (a sparse code). Montemurro et al found the average firing rate during the white noise stimulus to be 5.8 spikes per second and the average information per spike to be 5.3 bits/spike. By the standards of a conventional spike count code, this is a large amount of information (see Rieke et al. 1997); thus indicating that, in the time domain, the coding is sparse.

A third way to probe the coding of time-dependent stimuli uses the linear reconstruction method of Bialek et al. (1991). The reconstruction method assumes that the "meaning” of each spike is accurately expressed by its reverse correlation function (cross-correlation between stimulus and spike train). If this assumption is true, it should be possible to decode the spike train by convolving it with the reverse correlation function.

Jones et al. (2004a) applied this approach to the trigeminal ganglion. They measured the spike trains of single neurons evoked by white noise whisker deflection and used the above reconstruction method to construct a predicted stimulus. They found the average correlation coefficient between real and predicted stimuli to be 0.66 . Given the known sensitivity of neurons to rapid whisker motion, Jones et al also investigated prediction of stimulus velocity and stimulus acceleration. They found average correlation coefficients increased to 0.76 . These results imply that the linear decoding model accounts for around $50 \%$ of the variance in the ganglion response. Considering the simplicity of the model, this is an impressive result. Consistent with the work of Montemurro et al. on VPM neurons, it indicates that individual, subcortical neurons convey a considerable amount of information about a white noise whisker stimulus.

It was noted above that each subcortical whisker module consists only of around 200 neurons. From the point of view of the 'noisy neuron' hypothesis, it is mysterious how such a small neural circuit could transmit enough information to support the sophisticated texture processing of which rats are capable. In fact, functionally, the number of neurons per whisker is probably rather less than 200. Szwed et al. (2003), using the electrical whisking paradigm, found that $44 \%$ of ganglion cells fail to respond to a touch stimulus. (This probably reflects specific directional tuning, high activation thresholds and other factors). Thus, it seems likely that, for the lemniscal subcortical whisker pathway, the noisy neuron hypothesis is incorrect. Instead, given time-varying stimuli, neurons are reliable enough to convey large amounts of information by virtue of precise (sub-millisecond) timing of spikes. Thus, in a manner strikingly reminiscent of $\mathrm{H} 1$, these circuits can accomplish sophisticated sensory behaviours with perhaps $1 \%$ of the number of neurons available to cortex. 


\section{Mechanisms of coding by precise spike timing}

The studies of spike timing raise the question of how it is biophysically possible for neurons in the whisker system to employ high capacity spike timing codes. We argue that the answer can be traced to the work of Segundo. Bryant and Segundo (1976) recorded the response of aplysia neurons to injection of low-pass filtered white noise current. Their result was that the neuronal response is remarkably reproducible, and not at all the noisy result one might expect from the visual cortical literature. Bryant and Segundo accounted for the effect by a threshold model which fires a spike when the convolution of the stimulus with a given kernel (the average current leading up to a spike) passes threshold. They recognized that reliable spikes are triggered primarily by large, transient membrane currents. Given the capacitative nature of neuronal membrane, it is rapidly charged by such currents. Membrane potential passes quickly through threshold, so there is little opportunity for spike time to be affected by noise. The result is that trial-to-trial variation in spike time is minimal. More recent work has shown that properties of the active currents also contribute and are particularly important in explaining why, in contrast, neuronal responses to injection of even a large constant current are so noisy (Axmacher and Miles 2004; Azouz and Gray 2000; Gutkin et al. 2003).

We suggest that these ideas apply to coding in the whisker pathway in the following way. The stimuli for which spike timing has been shown to play an important role all include high velocity components - either, in the case of step deflections, at stimulus onset or, in the case of white noise and texture vibrations, throughout the stimulus. As discussed above, such stimuli evoke reliable and precisely timed spikes in trigeminal ganglion. Since mechanoreceptors are known to be velocity-sensitive, it is plausible that high velocity whisker motion leads to many ion channels opening in the mechanoreceptor membrane, thereby to a large membrane current and a reliable, precisely timed spike. The reason why this precision is preserved two synapses later at the level of the VPM thalamus, is probably due to the fact that the lemniscal synaptic input onto VPM cells is mediated by potent synapses (Brecht and Sakmann 2002; Castro-Alamancos 2002b; Deschenes et al. 2003). In this way, afferent spikes induce strong synaptic currents in VPM relay cells. By the Bryant-Segundo mechanism, these strong currents may induce rapid increase in VPM membrane potential and hence reliable spikes. In sum, we suggest that precise spike timing is to be expected to play an important role in coding in the subcortical whisker pathway whenever the stimuli to be discriminated differ in their high velocity components. We emphasise that this argument applies to the lemniscal pathway. Interestingly, the paralemniscal pathway through POm thalamus has very different properties. Here, in contrast to VPM, the afferent synapses are 
weak: whisker deflection response depends on feedback from barrel cortex (Diamond et al. 1992a) and is modulated by inhibition from the zona incerta (Trageser and Keller 2004). Perhaps as a consequence, POm responses exhibit much more jitter than VPM responses (Diamond et al. 1992b). This important difference is consistent with the proposal that the paralemniscal pathway has a distinct functional role in coding whisker position (Ahissar et al. 2000; for a different view, see Masri et al. 2008).

However, this account cannot, by itself, explain why precise spike timing is also evident in the responses of neurons in barrel cortex. The reason is that individual synapses from VPM relay cells onto layer 4 spiny stellate cells are much weaker than the subcortical synapses just discussed (Bruno and Sakmann 2006; Gil et al. 1999). In contrast to the subcortical circuit, a single thalamic spike is unlikely to reliably trigger a cortical spike. How then might it be possible for barrel cortical neurons to display coding by precise spike timing?

Because neurons at all levels of the pathway respond well to high velocity events, it has been suggested that rapid onset (high velocity) deflection of a given whisker causes robust, synchronous firing within the corresponding thalamic barreloid (Pinto et al. 2000). This in turn produces a near-synchronous volley of synaptic input onto neurons in the corresponding cortical barrel, and hence a transient, high amplitude excitatory synaptic current, resulting in a precisely timed cortical spike.

Conversely, lower-velocity sinusoidal stimuli do not significantly increase VPM synchrony compared to the spontaneous level (Bruno and Sakmann 2006). This raises the important question of how the comparatively weak thalamocortical input resulting from extended sinusoidal stimulation can successfully drive cortical responses. It is possible that intracortical amplification in layer 4 fulfils this role (Beierlein et al. 2002; Douglas et al. 1995), but the issue remains unresolved (see e.g., Alonso 2006).

\section{Decoding responses of barrel cortex neurons}

The finding of precise spike timing in barrel cortex indicates that cortical circuits do decode spike timing information conveyed by thalamic relay cells - at least in part by the mechanism discussed above. However, cortical circuitry is very different to subcortical circuitry in that it is massively recurrent. Is the information available in the responses of cortical neurons decoded by the decision-making circuits of the brain? A long-established method for addressing this issue is to train an animal to perform a sensory discrimination task, to induce firing in a neuronal population by passing current through an extracellular microelectrode and 
to test whether this influences the animal's choices. Remarkable new techniques have recently been developed, by means of which it is possible to selectively stimulate neurons. It has been found that, inducing a train of ca 14 spikes in a single neuron in barrel cortex can cause a statistically significant bias in a rodent's behaviour (Houweling and Brecht 2008). Conversely, rodents can report the occurrence of one induced spike in each of 100-400 barrel cortex neurons (Huber et al. 2008). Thus a volley of spikes from a single neuron is decodable, and a single spike from each neuron in a population is decodable. Whether spike timing is decodable has not yet been tested in barrel cortex, but has in other systems. In auditory cortex, rats can decode the relative timing of electrically induced activity in two neuronal populations, even when the difference is $3 \mathrm{~ms}$ (Yang et al. 2008). However, in a study of primate somatosensory cortex, no correlation between spike timing and behaviour was observed (Luna et al. 2005). This is an important topic for future investigation.

\section{Decoding spike timing information without an external clock}

The results reviewed above shows that spike timing has the potential to be important to cortical information transmission. However, it is important to consider whether these codes could be decoded, even in principle, by a downstream neural system. One reason this is an issue is that, unlike the experimenter, the animal has no independent knowledge about when each stimulus occurred (“external clock”). Some types of code are strongly clock-dependent. For example, the animal cannot know if the latency of a given neuron on a given trial was 5 ms or $10 \mathrm{~ms}$ (Fig. 4A-B). Given the prevalence of latency codes in sensory systems, this is an important case. Other types of code are not clock-dependent. A simple example is the interspike interval code (Fig. 4C-D). In practice, it may be far from intuitively obvious the extent to which a given spike timing code is clock-dependent or not. A crucial first step in addressing this issue is to quantify clock-dependency. Despite the importance of this question, surprisingly little attention has so far been devoted to it. In this section we review recent work developing a theoretical framework and applying it to the rat whisker system.

FIGURE 4 AROUND HERE

\section{Information theoretic framework for decoding}

The mutual information $I(S ; R)$ carried by a neural response, Equation 1, is an upper bound on the information that is available on an individual trial, to an observer. Shannon showed that 
there is an optimal decoding algorithm able to extract $I(S ; R)$ bits of information. In general, the optimal decoder needs to know perfectly the posterior probabilities $\mathrm{P}(\mathbf{s} \mid \mathbf{r})$. Observers with imperfect knowledge of $\mathrm{P}(\mathbf{s} \mid \mathbf{r})$ may not be able to decode as much information. How much information do we lose if we try to decode the neuronal response using posterior probabilities which are degraded due to imprecise knowledge of stimulus time?

To study in a parametric way the effect of degraded knowledge of the external clock, Arabzadeh et al. (2006) added uncertainty $\Delta t_{\text {external }}$ to the temporal alignment between response and stimulus time. They hypothesized that the imperfect decoder would base its interpretation of the current response $\mathbf{r}$ not on the correct posterior distribution $\mathrm{P}(\mathbf{s} \mid \mathbf{r})$, but on an approximate posterior distribution $\mathrm{Q}(\mathbf{s} \mid \mathbf{r})$ obtained by pooling the distribution of spike patterns $\mathbf{r}$ shifted within the range $\pm 1 / 2 \triangle t_{\text {external }}$. They then used information theory to quantify the information loss. Although there is no general closed-form expression for such an information loss (Merhav et al. 1994), Latham and Nirenberg (2005) recently derived a simple upper bound. Generalizing this work to the case of external clock degradation, Arabzadeh et al. derived an upper bound to the information lost when decoding using $Q(\mathbf{s} \mid \mathbf{r})$ rather than $\mathrm{P}(\mathbf{s} \mid \mathbf{r})$, termed $\Delta I_{\text {external: }}$

$$
\Delta I_{\text {external }}=\sum_{\mathbf{s}} \sum_{\mathbf{r}} \mathrm{P}(\mathbf{r}) \mathrm{P}(\mathbf{s} \mid \mathbf{r}) \log _{2} \frac{\mathrm{P}(\mathbf{s} \mid \mathbf{r})}{\mathrm{Q}(\mathbf{s} \mid \mathbf{r})}
$$

The quantity:

$$
I_{\text {external }}=I-\Delta I_{\text {external }}=\sum_{\mathbf{s}} \sum_{\mathbf{r}} \mathrm{P}(\mathbf{r}) \mathrm{P}(\mathbf{s} \mid \mathbf{r}) \log _{2} \frac{\mathrm{Q}(\mathbf{s} \mid \mathbf{r})}{\mathrm{P}(\mathbf{s})}
$$

is a lower bound to the information available to a decoder that operates on posteriors $\mathrm{Q}(\mathbf{s} \mid \mathbf{r})$ calculated with temporal precision $\Delta t_{\text {external }}$.

To understand more intuitively the meaning and the behavior of $I_{\text {external }}$ and its relationship with $I(S ; R)$, it is useful to consider the two simulated neurons illustrated in Fig. 4. The first simulated neuron fires single spikes with latency of $10 \mathrm{~ms}$ to stimulus 1 (Fig. 4A) and $20 \mathrm{~ms}$ to stimulus 2 (Fig. 4B). If we quantify the neural response $\mathbf{r}$ by measuring, with respect to the stimulus onset, the times of all spikes emitted in a 0-30 ms post-stimulus, the value of information $I(S: R)$ is 1 bit (which corresponds to $100 \%$ correct stimulus discrimination). However, this latency code clearly presupposes knowledge of the external clock. If the external clock uncertainty is large enough $\left(\Delta t_{\text {external }}>20 \mathrm{~ms}\right), \mathrm{Q}(\mathbf{s} \mid \mathbf{r})$ will equal $\mathrm{P}(\mathbf{s})$ (which means that the neural response provides no useful prediction about the stimulus) and $I_{\text {external }}$ drops to 0 bits. The second simulated neuron (Fig. 4C-D) encodes information by interspike 
intervals: $5 \mathrm{~ms}$ interspike intervals in response to stimulus 1 (Fig. 4C); $10 \mathrm{~ms}$ interspike intervals in response to stimulus 2 (Fig. 4D). In this case the information $I(S ; R)$ available from these spike times when there is a perfect knowledge of the external clock is 1 bit $(100 \%$ reliable stimulus discrimination). However, in contrast to the first example, $I_{\text {external }}$ does not drop at all and remains equal to 1 bit even if $\Delta t_{\text {external }}$ is large.

\section{Clock-dependent and clock independent coding in the whisker system}

Arabzadeh et al. (2006) used this information theoretic formalism to analyze responses of single neurons recorded from the trigeminal ganglion and of multi-unit clusters recorded from the barrel cortex of anaesthetized rats. The stimuli were 6 whisker vibration patterns induced by the objects described above. For ganglion, spike timing carries on average 1.5 bits of information when the stimulus time is known with perfect precision ( $\left.\Delta t_{\text {external }}=0 \mathrm{~ms}\right)$. For external clock precision $\Delta t_{\text {external }} \geq 50 \mathrm{~ms}, I_{\text {external }}$ computed from ganglion spike times saturates at 0.8 bits, which means that a large fraction (60\%) of ganglion timing information is clock robust. Importantly, the clock-robust spike timing information is markedly greater than the information available in the ganglion spike counts, which is 0.4 bits. Thus there is a real advantage in decoding ganglion spike timing rather than spike count, even when the stimulus time is not known. However, Arabzadeh et al. found different results for barrel cortex. When $\Delta t_{\text {external }}$ is $0 \mathrm{~ms}$, spike timing carries 0.45 bits of information, significantly more than that carried by spike count (0.3 bits). Yet, even a small imprecision in knowledge of stimulus time ( $\Delta t_{\text {external }} \geq 10 \mathrm{~ms}$ ) causes the cortical spike timing information to saturate at a value $I_{\text {external }}$ equal to the spike count information. Thus, the extra information available in cortical spike timing above and beyond that carried by spike counts is available to downstream neural circuits only when the stimulus time is known with high temporal precision.

Thus, there seems to be a transformation from "clock independent" coding in the periphery to "clock dependent" coding in cortex. We hypothesise that this is because the ganglion employs an inter-spike interval code whereas cortex employs a latency code - however, further work is necessary to test this. One potential advantage of such a transformation is that ganglion cells and their direct targets are unlikely to receive external clock information; thus they may be forced to deliver information in a clock independent way.

Two possibilities have been suggested for how decoders of the cortical signal might make use of spike timing information. First, an important characteristic of active sensory systems is that the nervous system is, in principle, capable of generating its own estimate of stimulus 
time. Since the collection of whisker data under natural conditions is an active process initiated by a motor command, decoding circuits might be able to use either the output of the motor system (Ahrens and Kleinfeld 2004) or sensory feedback (Szwed et al. 2003) to estimate stimulus time. Consistent with this possibility, evidence has been accumulating for a “whisking signal” in barrel cortex (Fee et al. 1997; Ganguly and Kleinfeld 2004). Thus, information carried by cortical spike timing, though clock-dependent, may be decoded by cortical neurons with access to whisking signals.

Second, the external clock problem can be very different at the population level, compared to the single neuron level. A population may encode information in a clock-independent way by the rank order of latencies (Gollisch and Meister 2008; Van Rullen and Thorpe 2001). For example, in barrel cortex, deflection of whisker D1 evokes spikes first in barrel D1, later in barrel D2, whereas deflection of whisker D2 evokes the opposite sequence of spikes. Hence, a simple hypothesis is that, whereas the single column code may be a clock-dependent latency code, the multi-column code may be a clock-independent inter-spike interval code. Spike timing relative to the activity of other neurons, or relative to fluctuations in the state of the cortical network which are reflected in local field potential fluctuations (Huxter et al. 2003; Montemurro et al. 2008), is a code that can be read without reference to any externally generated signals (Bullock 1993; Hopfield 1995). Thus, an important question for future research is how the robustness of the cortical code with respect to $\Delta t_{\text {external }}$ scales with the size of the neuronal population.

\section{Contextual effects on whisker representations}

Responses to a whisker stimulus are significantly affected by stimulation context, behavioural context, and internal context. In a natural setting, a whisker deflection seldom happens in either spatial or temporal isolation: 'stimulation context' refers to on-going stimulation of other whiskers and/or to the history of stimulation; 'behavioural context' to whether the stimulus is encountered passively or during active exploration; 'internal context' to fluctuations in the brain's activity level, characteristic of the animal's state of wakefulness and alertness. It is clear that evoked responses can be profoundly affected by these contextual factors, but little is known about how this specifically modifies whisker representations. In this section, we first discuss stimulation context, arguably the simplest form of context dependence and the one where the most evidence is available concerning effects on information encoding. We then briefly consider behavioural and internal context. 


\section{Stimulation context}

As mentioned above, the "classical" stimulus design for the whisker system consists of isolated discrete ramp-and-hold whisker deflections. These stimuli are temporally discrete in that, typically, successive repetitions are separated by at least $1 \mathrm{~s}$, and spatially discrete in that usually only a single whisker is deflected. Discrete whisker deflections comprise a small subset of the modes of stimulation that may be encountered by the system, and recent studies have begun to examine how stimulus encoding might be altered when stimuli are not discrete either in time or in space.

\section{Contextual effects in time: simple forms of interaction between stimulus parameters}

How is stimulus encoding affected if whisker movements are not discrete? In layer 4 of barrel cortex, spike timing precision for isolated stimuli arises due to a pattern of thalamocortical synaptic activation involving synchronous thalamic responses followed by tightly timed inhibition. This behaviour depends sensitively on the relative strength and timing of excitation and inhibition and on the ability of the postsynaptic membrane to identify inputs that arrive separately in time: these properties can change when stimuli are extended or repeated in time, altering responses in the postsynaptic neuron and also further up the pathway.

As an example, consider directional tuning. For neurons in the trigeminal ganglion, tuning is strong both for discrete stimuli and for "white noise" stimuli (Arabzadeh et al. 2005; Gibson and Welker 1983; Jones et al. 2004b; Zucker and Welker 1969). For neurons in later stages of the pathway, directional selectivity is weaker, and depends on the particular synaptic inputs received and their postsynaptic integration. In layer 4 of barrel cortex, discrete stimulation in the preferred direction evokes synaptic excitation that is large relative to inhibition and also has short latency, providing a fast-rising signal and a large "window of opportunity" for spiking; in other directions, discrete stimulation evokes excitation that is smaller relative to inhibition and that occurs with a smaller temporal advantage (Wilent and Contreras 2005). The net result is a higher probability of spiking in the preferred direction. However, for white noise stimuli cortical directional tuning is weaker (Arabzadeh et al. 2005; Hasenstaub et al. 2007; Maravall et al. 2007). A modeling study (Puccini et al. 2006) showed that, surprisingly, these two kinds of behavior can be reproduced by the same model neuron, with identical parameters, when driven by stimuli repeated at low rates (producing strong tuning) or high rates (weak tuning). At work here are general properties of synaptic integration. Integration time constants for neurons receiving thalamic input can range down to $\sim 1$ ms for discrete 
stimuli or at stimulus onset, but can be greater than $10 \mathrm{~ms}$ during prolonged stimulation (Gabernet et al. 2005). If stimulus repetition rates are low compared to the membrane integration time-scale, the postsynaptic neuron is able to detect volleys of excitation and inhibition as being separate, and directional tuning is strong. However, for stimuli containing high frequencies, the postsynaptic neuron may no longer be able to separate successive synaptic inputs, leading to an effective averaging out of inputs evoked by motion in different directions. In sum, changes in one stimulus parameter, repetition rate, may affect representation of another parameter, whisker direction.

\section{Contextual effects in time: adaptation}

Perhaps the most familiar aspect of neuronal response dynamics is that neurons accommodate to ongoing or repeated exposure to an environment (reviewed by Wark et al. 2007). As a consequence, the response to a particular stimulus depends on the recent history of stimulation. The term "adaptation” has been widely used in reference to any phenomenon that displays this property: it describes a family of behaviours that almost certainly reflect distinct underlying mechanisms and effects on information encoding. In the whisker system adaptation appears in at least three distinct forms.

\section{Adaptation to a constant stimulus}

In the first mode of adaptation (Fig. 5A), the response of most neurons in the whisker system to a maintained whisker deflection consists of an initial transient component that rapidly decays, and/or a sustained component that lasts as long as the stimulus. Neurons are usually classified as 'rapidly adapting' (RA) - no sustained response component - or as 'slowly adapting' (SA) - significant sustained component. Recent work in rats has reported functional effects of these categories (Stuttgen et al. 2006).

\section{Adaptation to repetitive stimuli}

In a second mode of adaptation (Fig. 5B), the response to each in a train of repeated whisker deflections decreases over time (Simons 1985). This form of adaptation filters out stimuli repeated at rates higher than a remarkably low cut-off, which in anesthetized animals is typically under $10 \mathrm{~Hz}$ (Ahissar et al. 2001; Ahissar et al. 2000; Castro-Alamancos 2002a, c; Castro-Alamancos and Oldford 2002; Garabedian et al. 2003; Webber and Stanley 2004). This frequency range overlaps with the normal whisking range $(5-25 \mathrm{~Hz})$, predicting that when an object is struck on successive whisks, the response on each cycle will markedly 
decrease unless the whisking frequency is at the low end of its range $\leqslant 5 \mathrm{~Hz}$ ) (Garabedian et al. 2003). However, this does not mean that neurons cannot encode high frequency stimulus components: as discussed above, barrel cortical neurons reliably encode whisker vibrations whose power differs in the 20-150 Hz range (Arabzadeh et al. 2005; Hipp et al. 2006). Adaptation to repetitive stimuli has a number of interesting properties. (1) Its extent increases with deeper levels of anaesthesia (compare Garabedian et al. 2003; Khatri et al. 2004) and is modulated during behaviour (see below). (2) Its extent and time course are different at successive stages of the whisker system (Castro-Alamancos 2002a, c; Castro-Alamancos and Oldford 2002; Chung et al. 2002; Diamond et al. 1992b; Fraser et al. 2006; Khatri et al. 2004; Sosnik et al. 2001). More central stages typically undergo greater adaptation and do so at lower frequencies. (3) Responses adapt differently across the lemniscal and paralemniscal pathways and, due to the segregation of pathways into different cortical layers, also across layers. In the lemniscal pathway, response magnitudes decrease in the adapted steady state, but there is no change in latency or loss of temporal precision in subcortical stages. Conversely, in the paralemniscal pathway, latencies increase and temporal precision is degraded in the adapted steady state (Ahissar et al. 2001; Ahissar et al. 2000; Derdikman et al. 2006; Sosnik et al. 2001), but see (Masri et al. 2008). However, cortical paralemniscal firing rates increase with increasing frequency, providing a code for whisking repetition frequency (Melzer et al. 2006). Differences in adaptation across the lemniscal and paralemniscal pathways may therefore have a functional correlate in the different modes of whisker encoding postulated for the two pathways (Ahissar et al. 2001; Ahissar et al. 2000): reviewed by Alloway (2008). (4) Adaptation to repetitive stimulation can sharpen neuronal tuning properties on successive whisks, including both whisker selectivity (Katz et al. 2006) and tuning to whisker direction (Khatri and Simons 2007). (5) In an interesting instance of sensitivity to noise, regular-spiking (putative excitatory) cortical neurons adapt more weakly to stimuli repeated at irregular intervals than to stimuli repeated at a constant rate (Lak et al. 2008).

The principal mechanism of cortical adaptation to repetitive stimulation is short-term synaptic depression (Chung et al. 2002). These changes in synaptic drive are typically fast: in barrel cortex, Webber and Stanley (2004; 2006) have shown that post-excitatory suppression following an isolated vibrissa deflection already predicts how the response will adapt to a long sequence of deflections. 


\section{Adaptation to stimulus statistics}

A third form of adaptation reflects an adjustment of neuronal stimulus-response relationships to better match the stimulus statistics of the current sensory environment. Such an adjustment can improve information transmission, giving neurons the ability to resolve individual stimulus values irrespective of background. Neurons in the whisker system also adapt in this sense (Fig. 5C) (Garcia-Lazaro et al. 2007; Maravall et al. 2007). Adapting neurons in the barrel cortex adjust their coding properties by rescaling their sensitivity according to the the current distribution of whisker amplitude and velocity (Maravall et al. 2007). This rescaling maintains the information that spikes convey about stimulus features in the face of major changes in stimulus dynamic range (Diaz-Quesada and Maravall 2008; Maravall et al. 2007).

While synaptic dynamics are the dominant mechanism of adaptation to repetitive stimulation, intrinsic properties account for the main properties of adaptation to stimulus statistics. In the barrel cortex, adaptation to stimulus statistics correlates with the amplitude of calciumdependent slow hyperpolarizations. This form of adaptation is thus highly stimulus specific, as it depends on the ability of a stimulus to activate comparatively slow membrane mechanisms (Diaz-Quesada and Maravall 2008).

In sum, the three modes of adaptation in the whisker system depend on distinct mechanisms, recruited differentially by the form of stimulation.

\section{Spatial context}

Another type of contextual effect involves interactions across whiskers. When a whisker is displaced together with its neighbours, as is bound to occur in natural settings, the response is different from that elicited by the whisker displacement in isolation (Simons 1985). The cellular basis is probably that responses elicited by both principal and neighbouring whiskers typically consist of a characteristic excitation-inhibition pattern (Higley and Contreras 2003; Moore and Nelson 1998). This implies that neighbouring whiskers can potentiate or inhibit each other's responses if stimulated with the appropriate temporal offset. Intriguingly, synaptic inputs from a non-principal whisker may be "silent": that is, they may be subthreshold for spike initiation, and only reveal itself when the whisker is stimulated together with the principal one, inhibiting the response (Simons and Carvell 1989). These "shunting" interactions are analogous to visual phenomena known as "extra-classical receptive field effects”, which concern the influence of stimuli presented outside the receptive field. 
Interference between whiskers varies depending on the identity, number and arrangement of the stimulated whiskers (Drew and Feldman 2007; Ego-Stengel et al. 2005; Mirabella et al. 2001; Simons 1985), the time delay between them (Erchova et al. 2003; Goldreich et al. 1998; Shimegi et al. 2000; Shimegi et al. 1999; Simons 1985), the angular directions of whisker motion (Kida et al. 2005; Shimegi et al. 2000; Simons 1985), whether single stimuli or trains of stimuli are applied - as well as the train frequency - (Ego-Stengel et al. 2005), the recorded neuron's layer (Ego-Stengel et al. 2005; Ghazanfar and Nicolelis 1997; Shimegi et al. 1999) and excitatory or inhibitory nature (Brumberg et al. 1996). The parameter space is enormous and a thorough picture is hard to achieve, but it appears that under most conditions interactions are suppressive. Analysis of the effects of multi-whisker interactions on information encoding remains an open field.

\section{Behavioural context}

Most sensory systems are active: the visual information that enters our brain is controlled by saccadic eye movements, the tactile information that enters a rat's brain by how it moves its whiskers. Understanding how the response to whisker stimulation is affected by active sensing was long hampered by inability to monitor whisker motion in awake animals. However, recent advances in whisker tracking technology, together with the advent of several ingenious experimental designs in head-fixed and behaving animals (Bermejo et al. 1996; Bermejo et al. 1998; Bermejo et al. 2004; Harvey et al. 2001; Hentschke et al. 2006; Krupa et al. 2001; von Heimendahl et al. 2007), have begun to provide insight. There is wide agreement that responses to active sensing can be different to responses to passive stimulation and, in particular, that active behaviour tends to suppress artificially evoked responses (Chapin and Woodward 1981, 1982; Fanselow and Nicolelis 1999). Hentschke et al. (2006), using awake head-fixed rats trained to contact a moving object, measured deep-layer spiking responses to whisker contact. They found that when contact is passive, response to contact is large and widely distributed across cortex: when contact is active, the response is small and narrowly distributed, and rides on top of an elevated firing level that is specific to whisking bouts. In recordings of collective synaptic activity (Ferezou et al. 2006) and of neuronal membrane potential (Crochet and Petersen 2006), responses to passive stimulation during quiet wakefulness are large while responses to passive stimulation during whisking are much smaller. However, responses to contact with an object encountered during active whisking are again very large, and can propagate throughout most of barrel cortex. In these studies, the change in responsiveness during whisking appears to be centrally generated and not a direct 
result of activation of follicle mechanoreceptors. Indeed, there is abundant evidence that barrel cortex activity during active whisking increases and contains a motor signal: (1) Responses to repetitive stimulation adapt much less under active exploration than passive stimulation (Castro-Alamancos 2004; Crochet and Petersen 2006; Fanselow and Nicolelis 1999), possibly because motor-related activity puts synapses in a “pre-adapted” steady-state mode. Depending on cortical layer, some synapses even facilitate during active whisking (Derdikman et al. 2006). (2) Whisking correlates tightly with oscillatory components in single-unit activity (Fee et al. 1997) and in local field potentials (Ahrens and Kleinfeld 2004); the correlation is conserved even after transection of the nerve carrying direct sensory input (Ahrens and Kleinfeld 2004). (3) Awake whole-cell recordings (Crochet and Petersen 2006) demonstrate that the membrane potential of barrel cortex neurons is time-locked to whisker motion. Taken together, these findings indicates that responses to active touch contain information about whisker motion as well as stimulus identity. Furthermore, responses to active touch are less sparse (involve a greater number of spikes) than responses to passive stimulation, providing potentially larger bandwidth for conjoint encoding of motor and sensory information but also doubtless altering the meaning of the spiking codes described in earlier sections of this review. Exactly how the motor and sensory signals are jointly encoded, and how they may be unambiguously decoded, remains an open matter.

Recent work by von Heimendahl et al. (2007) reveals, however, that spike counts remain informative about texture identity in awake animals performing a discrimination task. In barrel cortex, rough textures evoke a $10 \%$ higher average firing rate than smooth textures. On average, the mutual information between stimulus identity and a multiunit cluster's firing rate was 0.03 bits on correct trials and 0.021 bits averaged over all trials (including ones where the animal made an incorrect discrimination). These values are much smaller than in previous work using anesthetized animals (Arabzadeh et al. 2005), presumably because of the much greater variability in experimental conditions in the awake freely moving animal (distance of head to texture; posture and angle of approach; properties of whisking motion). Information between stimulus identity and the animal's behavioural response was 0.46 bits, so each neuronal cluster carried in its firing rate about $4.6 \%$ of the total information used by the animal. This implies that either (1) spike count is the relevant code for tactile discrimination during active exploration but needs to be pooled across 20 (independent) neurons, (2) fewer neurons underlie the decision but other elements of the response, such as spike timing, are more informative, (3) other neurons than the recorded ones underlie the discrimination. 


\section{Internal context}

Responses to a given stimulus can be highly variable across different trials even under identical experimental conditions, particularly in the cortex. From the point of view of the experimenter, this variability is 'noise' and it might seem to indicate that neuronal responses are unreliable. However, another possibility is that the variability reflects internal processing states of the animal's brain. Consistent with this view, evidence has emerged that much of the cortical response variability is attributable to fluctuations in activity shared by a large fraction of neurons in a region (Anderson et al. 2000; Arieli et al. 1996; Azouz and Gray 1999; Deweese and Zador 2004; Lampl et al. 1999; Petersen et al. 2003). In the barrel cortex, activity fluctuations strongly affect sensory responses (Castro-Alamancos 2004; Erchova et al. 2002; Hasenstaub et al. 2007; Petersen et al. 2003; Reig and Sanchez-Vives 2007; Sachdev et al. 2004). These data suggest that apparently noisy sensory responses might, given the internal context, actually be reliable.

Much research has studied the relationship between internal state and sensory responses. The synaptic circuits providing sensory and spontaneous inputs to a barrel cortex neuron overlap extensively (e.g., Petersen et al. 2003; Sachdev et al. 2004). Moreover, spontaneous and stimulus-evoked activity can be very similar (MacLean et al. 2005; Petersen et al. 2003). There is thus a close relationship between evoked and spontaneous activity, and sufficiently strong stimuli can trigger transitions between low-activity and high-activity states (Hasenstaub et al. 2007; Reig and Sanchez-Vives 2007). There has been some controversy as to whether responsiveness to isolated whisker stimuli is increased or reduced when a neuron is in the high-activity state (Hasenstaub et al. 2007; Petersen et al. 2003; Reig and SanchezVives 2007; Sachdev et al. 2004). Responsiveness to time-varying stimuli is higher in the high-activity situation, at the cost of reduced response specificity and temporal precision (Hasenstaub et al. 2007). Fluctuations in activity also affect adaptation: states of high network activation can cause synapses to be "pre-adapted”, or tonically depressed (CastroAlamancos 2004; Castro-Alamancos and Oldford 2002), although other evidence suggests that activated synapses can also remain strong (Reig et al. 2006; Reig and Sanchez-Vives 2007). In sum, the net effects of fluctuations in activity on whisker information encoding are not fully understood; this will require trial-by-trial analyses that fully take into account response variability. We propose that information theory may be ideally suited to this important task (Montemurro et al. 2008). 


\section{Conclusions}

Application of information theoretic methods indicates that spike timing plays a major role in coding whisker information. This conclusion, first shown with simple, discrete whisker stimuli has now been shown to apply also to more complex and naturalistic whisker vibration patterns. Recent work has also borne out the earlier finding that sensory information processing in the whisker system is extremely rapid, with cortical information, even about complex temporally extended stimuli, peaking within 20-30 ms of stimulus onset.

Subcortically, in the lemniscal pathway, spike timing is extremely precise (sub-millisecond). This means that subcortical whisker neurons can transmit sensory information at remarkably high bandwidth. This offers a plausible hypothesis for how it is possible for the relatively small subcortical modules, consisting of only 100-200 neurons, to nonetheless transmit the large amount of information required to support the sophisticated sensory-guided behaviour of which rats are capable. This suggests that a reasonable working model for the mode of subcortical whisking processing is the 'high reliability, low noise' mode, first established in the invertebrate.

Coding in barrel cortex also appears to exhibit an important role for spike timing. Although reliability is high for some neurons, in general, it appears to be markedly less than subcortical reliability. However, this does not necessarily mean that cortex is noisy. An important component of cortical response variability is due to fluctuations in the state of the cortical network. Thus, it may be that cortical coding, given the context of its internal state, is in fact reliable. Testing this hypothesis is an important aim for future research.

Not all the information conveyed by a given neural code is necessarily used by downstream neural circuits. The problem of how information is passed across systems in the brain remains an important open question of current research. Here, we have discussed the problem by considering whether information in spike times can be used by downstream neural circuits that have limited knowledge of the 'external clock' - that is, the precise times at which the experimenter delivers each stimulus. We have considered two possible solutions. First, some types of neural code, for example, inter-spike interval codes, may be intrinsically clockrobust. Second, there is experimental evidence for efference copy signals being available in cortex. Future work, particularly of coding at the population level, will be necessary to resolve the decoding issue.

Recently, there has been intensive investigation of other types of contextual effects on responses in the whisker pathway. Another crucial topic for future research will be to link 
this work on contextual modulation to the information theoretic studies of coding discussed above. 


\section{Figure captions}

Figure 1. Arrangement of whiskers on the snout and correspondence with barrels in primary somatosensory cortex (SI). A. Approximate location of whisker follicles and their row (letters) and arc (numbers) coordinates. (Smallest rostral whiskers in C-E rows not shown). B. Barrels, as seen in horizontal section through layer IV of SI.

Figure 2. Sub-millisecond spike timing precision in the trigeminal ganglion. A. Spikes fired in response to 45 repetitions of a white noise whisker deflection stimulus, recorded in vivo, under urethane anaesthesia. B. Expanded view of one firing episode from panel A. Data recorded by R.S. Petersen and M.R. Bale.

Figure 3. Quantification of a neuronal response. Simulated response to 8 repetitions of two stimuli (upper and lower panels respectively). A. Times of spikes evoked by stimulus 1 , relative to stimulus onset. Time units arbitrary. B. Spike count on each trial. C. Spike timing on each trial (response window divided into 4 bins). D-E. Corresponding data for stimulus 2.

Figure 4. Dependence of spike timing codes on external clock. A-B. Spikes fired by a simulated neuron that encodes information by latency: $10 \mathrm{~ms}$ for stimulus 1 (panel A) and 20 ms for stimulus 2 (panel B). C-D. Spikes fired by a second simulated neuron that encodes information by interspike interval: 5 ms for stimulus 1 (panel C); $10 \mathrm{~ms}$ for stimulus 2 (panel D).

Figure 5. Adaptation in the whisker system. A. Adaptation to constant stimulus (top). Slow adapting (SA) neurons continue to respond as long as the stimulus lasts (middle), whereas rapidly adapting (RA) neurons respond only to its onset (bottom). B. Adaptation to a repetitive stimulus (top). A non-adapting ganglion-type response (middle), compared to an adapting cortex-type response (bottom). C. Adaptation to a white noise stimulus consisting of alternating low and high variance epochs (top). The tuning curve to velocity is measured separately for each epoch type. The tuning curve of a non-adapting neuron (middle) is the same under high and low variance conditions. That of an adapting neuron (bottom) rescales to the variance. All units arbitrary. 


\section{References}

Adrian ED. The impulses produced by sensory nerve endings: Part I. J Physiol 61: 49-72, 1926.

Ahissar E, Sosnik R, Bagdasarian K, and Haidarliu S. Temporal frequency of whisker movement. II. Laminar organization of cortical representations. J Neurophysiol 86: 354-367, 2001.

Ahissar E, Sosnik R, and Haidarliu S. Transformation from temporal to rate coding in a somatosensory thalamocortical pathway. Nature 406: 302-306, 2000.

Ahrens KF and Kleinfeld D. Current flow in vibrissa motor cortex can phase-lock with exploratory rhythmic whisking in rat. J Neurophysiol 92: 1700-1707, 2004.

Alloway KD. Information processing streams in rodent barrel cortex: the differential functions of barrel and septal circuits. Cereb Cortex 18: 979-989, 2008.

Alonso JM. Neuroscience. Neurons find strength through synchrony in the brain. Science 312: 1604-1605, 2006.

Andermann ML, Ritt J, Neimark MA, and Moore CI. Neural correlates of vibrissa resonance; band-pass and somatotopic representation of high-frequency stimuli. Neuron 42: 451-463, 2004.

Anderson JS, Lampl I, Gillespie DC, and Ferster D. The contribution of noise to contrast invariance of orientation tuning in cat visual cortex. Science 290: 1968-1972, 2000.

Arabzadeh E, Panzeri S, and Diamond ME. Deciphering the spike train of a sensory neuron: counts and temporal patterns in the rat whisker pathway. $J$ Neurosci 26: 9216-9226, 2006.

Arabzadeh E, Panzeri S, and Diamond ME. Whisker vibration information carried by rat barrel cortex neurons. J Neurosci 24: 6011-6020, 2004.

Arabzadeh E, Petersen RS, and Diamond ME. Encoding of whisker vibration by rat barrel cortex neurons: implications for texture discrimination. J Neurosci 23: 9146-9154, 2003.

Arabzadeh E, Zorzin E, and Diamond ME. Neuronal encoding of texture in the whisker sensory pathway. PLoS Biol 3: e17, 2005.

Arieli A, Sterkin A, Grinvald A, and Aertsen A. Dynamics of ongoing activity: explanation of the large variability in evoked cortical responses. Science 273: 1868-1871, 1996.

Attwell $D$ and Laughlin SB. An energy budget for signaling in the grey matter of the brain. $J$ Cereb Blood Flow Metab 21: 1133-1145, 2001.

Axmacher $\mathbf{N}$ and Miles R. Intrinsic cellular currents and the temporal precision of EPSPaction potential coupling in CA1 pyramidal cells. J Physiol 555: 713-725, 2004.

Azouz R and Gray CM. Cellular mechanisms contributing to response variability of cortical neurons in vivo. J Neurosci 19: 2209-2223, 1999.

Azouz R and Gray CM. Dynamic spike threshold reveals a mechanism for synaptic coincidence detection in cortical neurons in vivo. Proc Natl Acad Sci U S A 97: 8110-8115, 2000 .

Beierlein M, Fall CP, Rinzel J, and Yuste R. Thalamocortical bursts trigger recurrent activity in neocortical networks: layer 4 as a frequency-dependent gate. $J$ Neurosci 22: 98859894, 2002.

Belford GR and Killackey HP. Vibrissae representation in subcortical trigeminal centers of the neonatal rat. J Comp Neurol 183: 305-321, 1979.

Bermejo R, Harvey M, Gao P, and Zeigler HP. Conditioned whisking in the rat. Somatosens Mot Res 13: 225-233, 1996.

Bermejo R, Houben D, and Zeigler HP. Optoelectronic monitoring of individual whisker movements in rats. J Neurosci Methods 83: 89-96, 1998. 
Bermejo R, Szwed M, Friedman W, Ahissar E, and Zeigler HP. One whisker whisking: unit recording during conditioned whisking in rats. Somatosens Mot Res 21: 183-187, 2004.

Bezdudnaya $\mathbf{T}$ and Keller A. Laterodorsal nucleus of the thalamus: A processor of somatosensory inputs. J Comp Neurol 507: 1979-1989, 2008.

Bialek W, Rieke F, de Ruyter van Steveninck RR, and Warland D. Reading a neural code. Science 252: 1854-1857, 1991.

Brecht M. Barrel cortex and whisker-mediated behaviors. Curr Opin Neurobiol 17: 408-416, 2007.

Brecht $\mathbf{M}$ and Sakmann B. Whisker maps of neuronal subclasses of the rat ventral posterior medial thalamus, identified by whole-cell voltage recording and morphological reconstruction. J Physiol 538: 495-515, 2002.

Brumberg JC, Pinto DJ, and Simons DJ. Spatial gradients and inhibitory summation in the rat whisker barrel system. J Neurophysiol 76: 130-140, 1996.

Bruno RM and Sakmann B. Cortex is driven by weak but synchronously active thalamocortical synapses. Science 312: 1622-1627, 2006.

Bryant HL and Segundo JP. Spike initiation by transmembrane current: a white-noise analysis. J Physiol 260: 279-314, 1976.

Bullock TH. Integrative systems research on the brain: resurgence and new opportunities. Annu Rev Neurosci 16: 1-15, 1993.

Carvell GE and Simons DJ. Biometric analyses of vibrissal tactile discrimination in the rat. J Neurosci 10: 2638-2648, 1990.

Carvell GE and Simons DJ. Task- and subject-related differences in sensorimotor behavior during active touch. Somatosens Mot Res 12: 1-9, 1995.

Castro-Alamancos MA. Absence of rapid sensory adaptation in neocortex during information processing states. Neuron 41: 455-464, 2004.

Castro-Alamancos MA. Different temporal processing of sensory inputs in the rat thalamus during quiescent and information processing states in vivo. $J$ Physiol 539: 567-578, 2002a.

Castro-Alamancos MA. Properties of primary sensory (lemniscal) synapses in the ventrobasal thalamus and the relay of high-frequency sensory inputs. J Neurophysiol 87: 946953, 2002b.

Castro-Alamancos MA. Role of thalamocortical sensory suppression during arousal: focusing sensory inputs in neocortex. $J$ Neurosci 22: 9651-9655, 2002c.

Castro-Alamancos MA and Oldford E. Cortical sensory suppression during arousal is due to the activity-dependent depression of thalamocortical synapses. J Physiol 541: 319-331, 2002.

Chapin JK and Woodward DJ. Modulation of sensory responsiveness of single somatosensory cortical cells during movement and arousal behaviors. Exp Neurol 72: 164178, 1981.

Chapin JK and Woodward DJ. Somatic sensory transmission to the cortex during movement: gating of single cell responses to touch. Exp Neurol 78: 654-669, 1982.

Chung S, Li X, and Nelson SB. Short-term depression at thalamocortical synapses contributes to rapid adaptation of cortical sensory responses in vivo. Neuron 34: 437-446, 2002.

Cover TM and Thomas JA. Elements of information theory. New York: Wiley, 1991.

Crochet $\mathbf{S}$ and Petersen CC. Correlating whisker behavior with membrane potential in barrel cortex of awake mice. Nat Neurosci 9: 608-610, 2006.

de Ruyter van Steveninck RR, Lewen GD, Strong SP, Koberle R, and Bialek W. Reproducibility and variability in neural spike trains. Science 275: 1805-1808, 1997.

Derdikman D, Yu C, Haidarliu S, Bagdasarian K, Arieli A, and Ahissar E. Layer-specific touch-dependent facilitation and depression in the somatosensory cortex during active whisking. J Neurosci 26: 9538-9547, 2006. 
Deschenes M, Timofeeva E, and Lavallee $\mathbf{P}$. The relay of high-frequency sensory signals in the Whisker-to-barreloid pathway. J Neurosci 23: 6778-6787, 2003.

Deweese MR and Zador AM. Shared and private variability in the auditory cortex. $J$ Neurophysiol 92: 1840-1855, 2004.

Diamond ME, Armstrong-James M, Budway MJ, and Ebner FF. Somatic sensory responses in the rostral sector of the posterior group (POm) and in the ventral posterior medial nucleus (VPM) of the rat thalamus: dependence on the barrel field cortex. J Comp Neurol 319: 66-84, 1992a.

Diamond ME, Armstrong-James M, and Ebner FF. Somatic sensory responses in the rostral sector of the posterior group (POm) and in the ventral posterior medial nucleus (VPM) of the rat thalamus. J Comp Neurol 318: 462-476, $1992 \mathrm{~b}$.

Diamond ME, von Heimendahl $\mathbf{M}$, and Arabzadeh E. Whisker-mediated texture discrimination. PLoS Biol 6: e220, 2008a.

Diamond ME, von Heimendahl M, Knutsen PM, Kleinfeld D, and Ahissar E. 'Where' and 'what' in the whisker sensorimotor system. Nat Rev Neurosci 9: 601-612, 2008b.

Diaz-Quesada $\mathbf{M}$ and Maravall $\mathbf{M}$. Intrinsic mechanisms for adaptive gain rescaling in barrel cortex. J Neurosci 28: 696-710, 2008.

Douglas RJ, Koch C, Mahowald M, Martin KA, and Suarez HH. Recurrent excitation in neocortical circuits. Science 269: 981-985, 1995.

Drew PJ and Feldman DE. Representation of moving wavefronts of whisker deflection in rat somatosensory cortex. J Neurophysiol 98: 1566-1580, 2007.

Ego-Stengel V, Mello e Souza T, Jacob V, and Shulz DE. Spatiotemporal characteristics of neuronal sensory integration in the barrel cortex of the rat. $J$ Neurophysiol 93: 1450-1467, 2005.

Erchova IA, Lebedev MA, and Diamond ME. Somatosensory cortical neuronal population activity across states of anaesthesia. Eur J Neurosci 15: 744-752, 2002.

Erchova IA, Petersen RS, and Diamond ME. Effect of developmental sensory and motor deprivation on the functional organization of adult rat somatosensory cortex. Brain Res Bull 60: 373-386, 2003.

Fanselow EE and Nicolelis MA. Behavioral modulation of tactile responses in the rat somatosensory system. J Neurosci 19: 7603-7616, 1999.

Fee MS, Mitra PP, and Kleinfeld D. Central versus peripheral determinants of patterned spike activity in rat vibrissa cortex during whisking. J Neurophysiol 78: 1144-1149, 1997.

Ferezou I, Bolea S, and Petersen CC. Visualizing the cortical representation of whisker touch: voltage-sensitive dye imaging in freely moving mice. Neuron 50: 617-629, 2006.

Foffani G, Tutunculer B, and Moxon KA. Role of spike timing in the forelimb somatosensory cortex of the rat. J Neurosci 24: 7266-7271, 2004.

Fraser G, Hartings JA, and Simons DJ. Adaptation of trigeminal ganglion cells to periodic whisker deflections. Somatosens Mot Res 23: 111-118, 2006.

Gabernet L, Jadhav SP, Feldman DE, Carandini M, and Scanziani M. Somatosensory integration controlled by dynamic thalamocortical feed-forward inhibition. Neuron 48: 315327, 2005.

Ganguly $\mathbf{K}$ and Kleinfeld $\mathbf{D}$. Goal-directed whisking increases phase-locking between vibrissa movement and electrical activity in primary sensory cortex in rat. Proc Natl Acad Sci U S A 101: 12348-12353, 2004.

Garabedian CE, Jones SR, Merzenich MM, Dale A, and Moore CI. Band-pass response properties of rat SI neurons. J Neurophysiol 90: 1379-1391, 2003.

Garcia-Lazaro JA, Ho SS, Nair A, and Schnupp JW. Shifting and scaling adaptation to dynamic stimuli in somatosensory cortex. Eur J Neurosci 26: 2359-2368, 2007.

Ghazanfar AA and Nicolelis MA. Nonlinear processing of tactile information in the thalamocortical loop. J Neurophysiol 78: 506-510, 1997. 
Gibson JM and Welker WI. Quantitative studies of stimulus coding in first-order vibrissa afferents of rats. 1. Receptive field properties and threshold distributions. Somatosens Res 1: 51-67, 1983.

Gil Z, Connors BW, and Amitai Y. Efficacy of thalamocortical and intracortical synaptic connections: quanta, innervation, and reliability. Neuron 23: 385-397, 1999.

Goldreich D, Peterson BE, and Merzenich MM. Optical imaging and electrophysiology of rat barrel cortex. II. Responses to paired-vibrissa deflections. Cereb Cortex 8: 184-192, 1998.

Gollisch $\mathbf{T}$ and Meister $\mathbf{M}$. Rapid neural coding in the retina with relative spike latencies. Science 319: 1108-1111, 2008.

Guic-Robles E, Valdivieso C, and Guajardo G. Rats can learn a roughness discrimination using only their vibrissal system. Behav Brain Res 31: 285-289, 1989.

Gutkin B, Ermentrout GB, and Rudolph M. Spike generating dynamics and the conditions for spike-time precision in cortical neurons. J Comput Neurosci 15: 91-103, 2003.

Hartmann MJ, Johnson NJ, Towal RB, and Assad C. Mechanical characteristics of rat vibrissae: resonant frequencies and damping in isolated whiskers and in the awake behaving animal. J Neurosci 23: 6510-6519, 2003.

Harvey MA, Bermejo R, and Zeigler HP. Discriminative whisking in the head-fixed rat: optoelectronic monitoring during tactile detection and discrimination tasks. Somatosens Mot Res 18: 211-222, 2001.

Hasenstaub A, Sachdev RN, and McCormick DA. State changes rapidly modulate cortical neuronal responsiveness. J Neurosci 27: 9607-9622, 2007.

Heil P. First-spike latency of auditory neurons revisited. Curr Opin Neurobiol 14: 461-467, 2004.

Hentschke H, Haiss F, and Schwarz C. Central signals rapidly switch tactile processing in rat barrel cortex during whisker movements. Cereb Cortex 16: 1142-1156, 2006.

Higley MJ and Contreras D. Nonlinear integration of sensory responses in the rat barrel cortex: an intracellular study in vivo. J Neurosci 23: 10190-10200, 2003.

Hipp J, Arabzadeh E, Zorzin E, Conradt J, Kayser C, Diamond ME, and Konig P. Texture signals in whisker vibrations. J Neurophysiol 95: 1792-1799, 2006.

Hopfield JJ. Pattern recognition computation using action potential timing for stimulus representation. Nature 376: 33-36, 1995.

Houweling AR and Brecht $\mathbf{M}$. Behavioural report of single neuron stimulation in somatosensory cortex. Nature 451: 65-68, 2008.

Huber D, Petreanu L, Ghitani N, Ranade S, Hromadka T, Mainen Z, and Svoboda K. Sparse optical microstimulation in barrel cortex drives learned behaviour in freely moving mice. Nature 451: 61-64, 2008.

Huxter J, Burgess N, and O'Keefe $\mathbf{J}$. Independent rate and temporal coding in hippocampal pyramidal cells. Nature 425: 828-832, 2003.

Johansson RS and Birznieks I. First spikes in ensembles of human tactile afferents code complex spatial fingertip events. Nat Neurosci 7: 170-177, 2004.

Jones LM, Depireux DA, Simons DJ, and Keller A. Robust temporal coding in the trigeminal system. Science 304: 1986-1989, 2004a.

Jones LM, Lee S, Trageser JC, Simons DJ, and Keller A. Precise temporal responses in whisker trigeminal neurons. J Neurophysiol 92: 665-668, 2004b.

Katz Y, Heiss JE, and Lampl I. Cross-whisker adaptation of neurons in the rat barrel cortex. J Neurosci 26: 13363-13372, 2006.

Khatri V, Hartings JA, and Simons DJ. Adaptation in thalamic barreloid and cortical barrel neurons to periodic whisker deflections varying in frequency and velocity. $J$ Neurophysiol 92: 3244-3254, 2004.

Khatri V and Simons DJ. Angularly nonspecific response suppression in rat barrel cortex. Cereb Cortex 17: 599-609, 2007. 
Kida H, Shimegi S, and Sato $\mathbf{H}$. Similarity of direction tuning among responses to stimulation of different whiskers in neurons of rat barrel cortex. J Neurophysiol 94: 20042018, 2005.

Kleinfeld D, Ahissar E, and Diamond ME. Active sensation: insights from the rodent vibrissa sensorimotor system. Curr Opin Neurobiol 16: 435-444, 2006.

Krupa DJ, Matell MS, Brisben AJ, Oliveira LM, and Nicolelis MA. Behavioral properties of the trigeminal somatosensory system in rats performing whisker-dependent tactile discriminations. J Neurosci 21: 5752-5763, 2001.

Lak A, Arabzadeh E, and Diamond ME. Enhanced response of neurons in rat somatosensory cortex to stimuli containing temporal noise. Cereb Cortex 18: 1085-1093, 2008.

Lampl I, Reichova I, and Ferster D. Synchronous membrane potential fluctuations in neurons of the cat visual cortex. Neuron 22: 361-374, 1999.

Land MF and Collett TS. Chasing Behavior of Houseflies (Fannia-Canicularis) Description and Analysis. J Comp Physiol 89: 331-357, 1974.

Land PW and Simons DJ. Metabolic and structural correlates of the vibrissae representation in the thalamus of the adult rat. Neurosci Lett 60: 319-324, 1985.

Latham PE and Nirenberg S. Synergy, redundancy, and independence in population codes, revisited. J Neurosci 25: 5195-5206, 2005.

Lee KJ and Woolsey TA. A proportional relationship between peripheral innervation density and cortical neuron number in the somatosensory system of the mouse. Brain Res 99: 349353, 1975.

Leiser SC and Moxon KA. Relationship between physiological response type (RA and SA) and vibrissal receptive field of neurons within the rat trigeminal ganglion. J Neurophysiol 95: 3129-3145, 2006.

Luna R, Hernandez A, Brody CD, and Romo R. Neural codes for perceptual discrimination in primary somatosensory cortex. Nat Neurosci 8: 1210-1219, 2005.

MacKay D and McCulloch WS. The limiting information capacity of a neuronal link. Bull Math Biophys 14: 127-135, 1952.

MacLean JN, Watson BO, Aaron GB, and Yuste R. Internal dynamics determine the cortical response to thalamic stimulation. Neuron 48: 811-823, 2005.

Mainen ZF and Sejnowski TJ. Reliability of spike timing in neocortical neurons. Science 268: 1503-1506, 1995.

Maravall M, Petersen RS, Fairhall AL, Arabzadeh E, and Diamond ME. Shifts in coding properties and maintenance of information transmission during adaptation in barrel cortex. PLoS Biol 5: e19, 2007.

Masri R, Bezdudnaya T, Trageser JC, and Keller A. Encoding of stimulus frequency and sensor motion in the posterior medial thalamic nucleus. J Neurophysiol 100: 681-689, 2008.

Melzer P, Champney GC, Maguire MJ, and Ebner FF. Rate code and temporal code for frequency of whisker stimulation in rat primary and secondary somatic sensory cortex. Exp Brain Res 172: 370-386, 2006.

Merhav N, Kaplan G, Lapidoth A, and Shamai S. On Information Rates for Mismatched Decoders. Ieee T Inform Theory 40: 1953-1967, 1994.

Mirabella G, Battiston S, and Diamond ME. Integration of multiple-whisker inputs in rat somatosensory cortex. Cereb Cortex 11: 164-170, 2001.

Montemurro MA, Panzeri S, Maravall M, Alenda A, Bale MR, Brambilla M, and Petersen RS. Role of precise spike timing in coding of dynamic vibrissa stimuli in somatosensory thalamus. J Neurophysiol 98: 1871-1882, 2007a.

Montemurro MA, Rasch MJ, Murayama Y, Logothetis NK, and Panzeri S. Phase-offiring coding of natural visual stimuli in primary visual cortex. Curr Biol 18: 375-380, 2008. 
Montemurro MA, Senatore R, and Panzeri S. Tight data-robust bounds to mutual information combining shuffling and model selection techniques. Neural Comput 19: 29132957, 2007b.

Moore CI and Nelson SB. Spatio-temporal subthreshold receptive fields in the vibrissa representation of rat primary somatosensory cortex. J Neurophysiol 80: 2882-2892, 1998.

Moore GP, Perkel DH, and Segundo JP. Statistical analysis and functional interpretation of neuronal spike data. Annu Rev Physiol 28: 493-522, 1966.

Neimark MA, Andermann ML, Hopfield JJ, and Moore CI. Vibrissa resonance as a transduction mechanism for tactile encoding. J Neurosci 23: 6499-6509, 2003.

Panzeri S, Petersen RS, Schultz SR, Lebedev M, and Diamond ME. The role of spike timing in the coding of stimulus location in rat somatosensory cortex. Neuron 29: 769-777, 2001.

Panzeri S, Pola G, and Petersen RS. Coding of sensory signals by neuronal populations: the role of correlated activity. Neuroscientist 9: 175-180, 2003.

Panzeri S and Schultz SR. A unified approach to the study of temporal, correlational, and rate coding. Neural Comput 13: 1311-1349, 2001.

Panzeri S, Senatore R, Montemurro MA, and Petersen RS. Correcting for the sampling bias problem in spike train information measures. J Neurophysiol 98: 1064-1072, 2007.

Peters A and Payne BR. Numerical relationships between geniculocortical afferents and pyramidal cell modules in cat primary visual cortex. Cereb Cortex 3: 69-78, 1993.

Petersen CC. The functional organization of the barrel cortex. Neuron 56: 339-355, 2007.

Petersen CC, Hahn TT, Mehta M, Grinvald A, and Sakmann B. Interaction of sensory responses with spontaneous depolarization in layer 2/3 barrel cortex. Proc Natl Acad Sci U S A 100: 13638-13643, 2003.

Petersen RS, Brambilla M, Bale MR, Alenda A, Panzeri S, Montemurro MA, and Maravall M. Diverse and temporally precise kinetic feature selectivity in the VPm thalamic nucleus. Neuron in press, 2008.

Petersen RS and Diamond ME. Spatial-temporal distribution of whisker-evoked activity in rat somatosensory cortex and the coding of stimulus location. J Neurosci 20: 6135-6143, 2000.

Petersen RS and Panzeri S. A case study of population coding: stimulus localisation in the barrel cortex. In: Computational Neuroscience: A Comprehensive Approach, edited by Feng J: CRC Press, 2003.

Petersen RS, Panzeri S, and Diamond ME. Population coding of stimulus location in rat somatosensory cortex. Neuron 32: 503-514, 2001.

Petersen RS, Panzeri S, and Diamond ME. The role of individual spikes and spike patterns in population coding of stimulus location in rat somatosensory cortex. Biosystems 67: 187193, 2002.

Pierret T, Lavallee P, and Deschenes $\mathbf{M}$. Parallel streams for the relay of vibrissal information through thalamic barreloids. J Neurosci 20: 7455-7462, 2000.

Pinto DJ, Brumberg JC, and Simons DJ. Circuit dynamics and coding strategies in rodent somatosensory cortex. J Neurophysiol 83: 1158-1166, 2000.

Puccini GD, Compte A, and Maravall M. Stimulus dependence of barrel cortex directional selectivity. PLoS ONE 1: e137, 2006.

Reig R, Gallego R, Nowak LG, and Sanchez-Vives MV. Impact of cortical network activity on short-term synaptic depression. Cereb Cortex 16: 688-695, 2006.

Reig R and Sanchez-Vives MV. Synaptic transmission and plasticity in an active cortical network. PLoS ONE 2: e670, 2007.

Rice FL, Mance A, and Munger BL. A comparative light microscopic analysis of the sensory innervation of the mystacial pad. I. Innervation of vibrissal follicle-sinus complexes. $J$ Comp Neurol 252: 154-174, 1986. 
Rice FL and Munger BL. A comparative light microscopic analysis of the sensory innervation of the mystacial pad. II. The common fur between the vibrissae. J Comp Neurol 252: 186-205, 1986.

Rieke F, Warland DK, de Ruyter van Steveninck RR, and Bialek W. Spikes: Exploring the neural code. Cambridge, MA: MIT Press, 1997.

Ritt JT, Andermann ML, and Moore CI. Embodied information processing: vibrissa mechanics and texture features shape micromotions in actively sensing rats. Neuron 57: 599613, 2008.

Sachdev RN, Ebner FF, and Wilson CJ. Effect of subthreshold up and down states on the whisker-evoked response in somatosensory cortex. J Neurophysiol 92: 3511-3521, 2004.

Shadlen MN and Newsome WT. Noise, neural codes and cortical organization. Curr Opin Neurobiol 4: 569-579, 1994.

Shimegi S, Akasaki T, Ichikawa T, and Sato H. Physiological and anatomical organization of multiwhisker response interactions in the barrel cortex of rats. J Neurosci 20: 6241-6248, 2000.

Shimegi S, Ichikawa T, Akasaki T, and Sato $\mathbf{H}$. Temporal characteristics of response integration evoked by multiple whisker stimulations in the barrel cortex of rats. J Neurosci 19: 10164-10175, 1999.

Simons DJ. Response properties of vibrissa units in rat SI somatosensory neocortex. $J$ Neurophysiol 41: 798-820, 1978.

Simons DJ. Temporal and spatial integration in the rat SI vibrissa cortex. J Neurophysiol 54: 615-635, 1985.

Simons DJ and Carvell GE. Thalamocortical response transformation in the rat vibrissa/barrel system. J Neurophysiol 61: 311-330, 1989.

Sosnik R, Haidarliu S, and Ahissar E. Temporal frequency of whisker movement. I. Representations in brain stem and thalamus. J Neurophysiol 86: 339-353, 2001.

Strong SP, Koberle R, de Ruyter van Steveninck R, and Bialek W. Entropy and information in neural spike trains. Phys Rev Lett 80: 197-200, 1998.

Stuttgen MC, Ruter J, and Schwarz C. Two psychophysical channels of whisker deflection in rats align with two neuronal classes of primary afferents. J Neurosci 26: 7933-7941, 2006.

Szwed M, Bagdasarian K, and Ahissar E. Encoding of vibrissal active touch. Neuron 40: 621-630, 2003.

Tolhurst DJ, Movshon JA, and Dean AF. The statistical reliability of signals in single neurons in cat and monkey visual cortex. Vision Res 23: 775-785, 1983.

Trageser JC and Keller A. Reducing the uncertainty: gating of peripheral inputs by zona incerta. J Neurosci 24: 8911-8915, 2004.

Urbain $\mathbf{N}$ and Deschenes $\mathbf{M}$. A new thalamic pathway of vibrissal information modulated by the motor cortex. J Neurosci 27: 12407-12412, 2007.

van der Loos H. Barreloids in Mouse Somatosensory Thalamus. Neuroscience Letters 2: 1-6, 1976.

Van Rullen $\mathbf{R}$ and Thorpe SJ. Rate coding versus temporal order coding: what the retinal ganglion cells tell the visual cortex. Neural Comput 13: 1255-1283, 2001.

von Heimendahl M, Itskov PM, Arabzadeh E, and Diamond ME. Neuronal activity in rat barrel cortex underlying texture discrimination. PLoS Biol 5: e305, 2007.

Wark B, Lundstrom BN, and Fairhall A. Sensory adaptation. Curr Opin Neurobiol 17: 423-429, 2007.

Webber RM and Stanley GB. Nonlinear encoding of tactile patterns in the barrel cortex. $J$ Neurophysiol 91: 2010-2022, 2004.

Webber RM and Stanley GB. Transient and steady-state dynamics of cortical adaptation. $J$ Neurophysiol 95: 2923-2932, 2006. 
Welker E and Van der Loos H. Quantitative correlation between barrel-field size and the sensory innervation of the whiskerpad: a comparative study in six strains of mice bred for different patterns of mystacial vibrissae. J Neurosci 6: 3355-3373, 1986.

Wilent WB and Contreras D. Dynamics of excitation and inhibition underlying stimulus selectivity in rat somatosensory cortex. Nat Neurosci 8: 1364-1370, 2005.

Woolsey TA and Van der Loos $\mathbf{H}$. The structural organization of layer IV in the somatosensory region (SI) of mouse cerebral cortex. The description of a cortical field composed of discrete cytoarchitectonic units. Brain Res 17: 205-242, 1970.

Yang Y, DeWeese MR, Otazu GH, and Zador AM. Millisecond-scale differences in neural activity in auditory cortex can drive decisions. Nat Neurosci 11: 1262-1263, 2008.

Zucker E and Welker WI. Coding of somatic sensory input by vibrissae neurons in the rat's trigeminal ganglion. Brain Res 12: 138-156, 1969. 
A

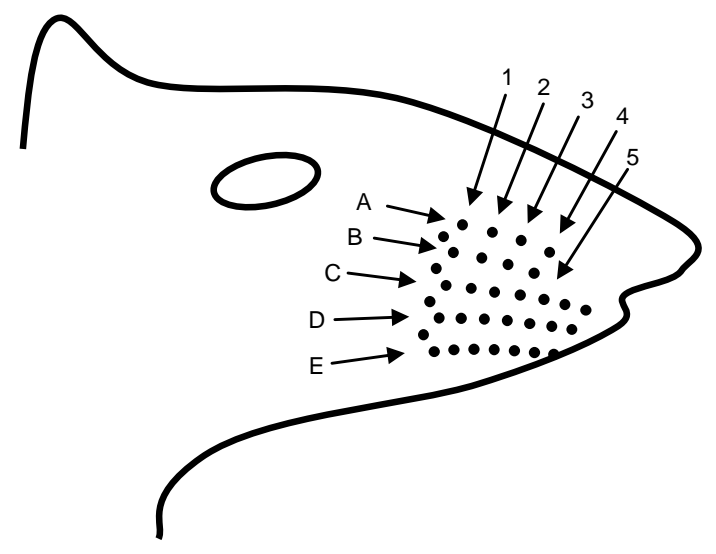

B

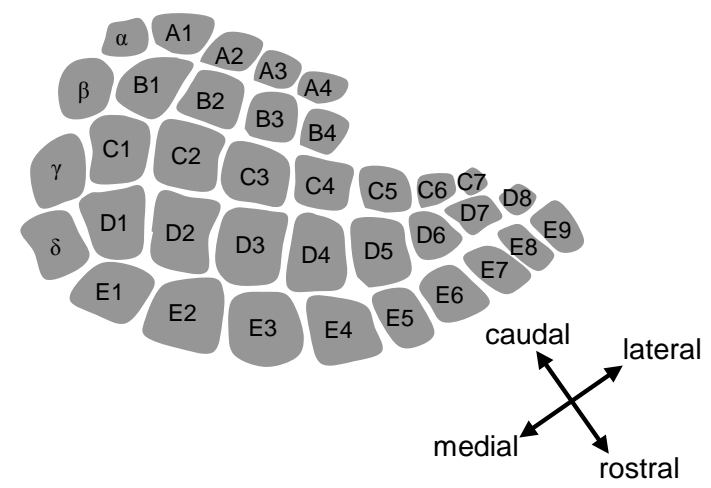

Figure 1. Arrangement of whiskers on the snout and correspondence with barrels in primary somatosensory cortex (SI). A. Approximate location of whisker follicles and their row (letters) and arc (numbers) coordinates. (Smallest rostral whiskers in C-E rows not shown). B. Barrels, as seen in horizontal section through layer IV of SI. 

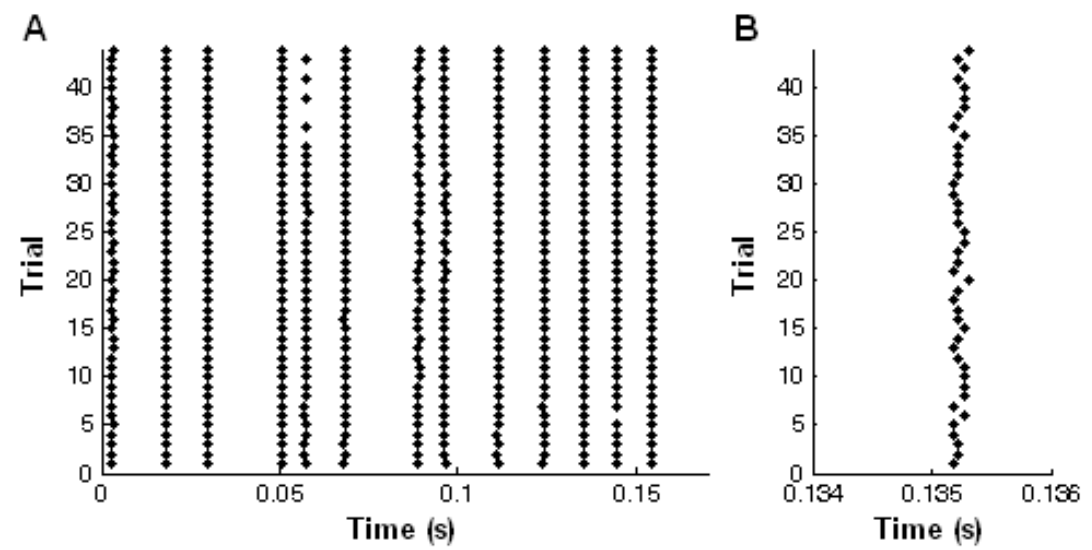

Figure 2. Sub-millisecond spike timing precision in the trigeminal ganglion. A. Spikes fired in response to 45 repetitions of a white noise whisker deflection stimulus, recorded in vivo, under urethane anaesthesia. B. Expanded view of one firing episode from panel A. Data recorded by R.S. Petersen and M.R. Bale. 


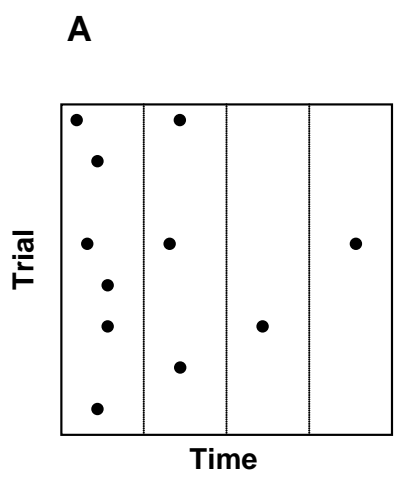

D

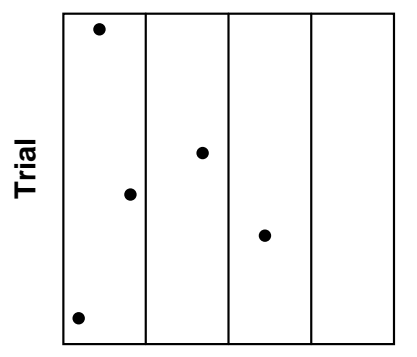

Time
B

Spike count

2

1

0

3

1

2

1

C

Spike timing

1100

1000

0000

1101

1000

1010

0100

1000

$\begin{array}{ll}\text { E } & \text { F } \\ \text { Spike count } & \text { Spike timing }\end{array}$

1000

0000

0000

0100

1000

0010

0000

1000

Figure 3. Quantification of a neuronal response. Simulated response to 8 repetitions of two stimuli (upper and lower panels respectively). A. Times of spikes evoked by stimulus 1 , relative to stimulus onset. Time units arbitrary. B. Spike count on each trial. C. Spike timing on each trial (response window divided into 4 bins). D-E. Corresponding data for stimulus 2. 

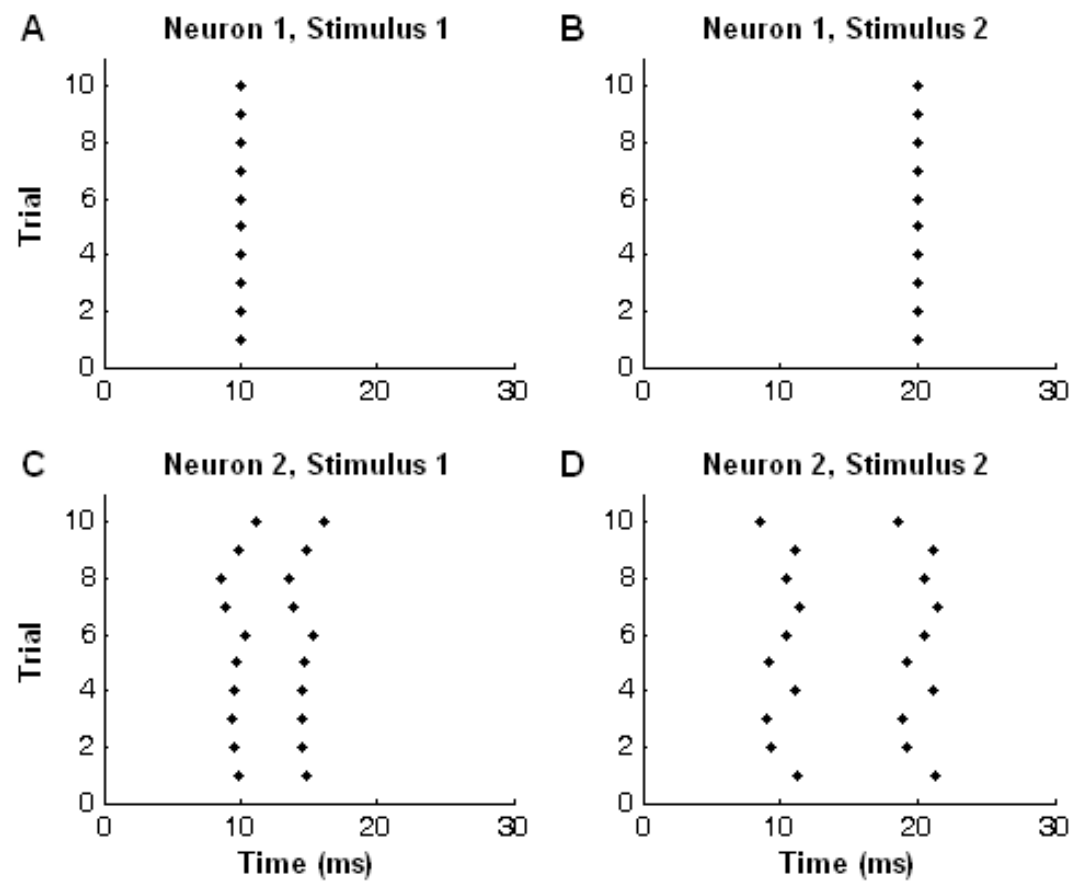

Figure 4. Dependence of spike timing codes on external clock. A-B. Spikes fired by a simulated neuron that encodes information by latency: $10 \mathrm{~ms}$ for stimulus 1 (panel A) and $20 \mathrm{~ms}$ for stimulus 2 (panel B). C-D. Spikes fired by a second simulated neuron that encodes information by interspike interval: $5 \mathrm{~ms}$ for stimulus 1 (panel C); $10 \mathrm{~ms}$ for stimulus 2 (panel D). 

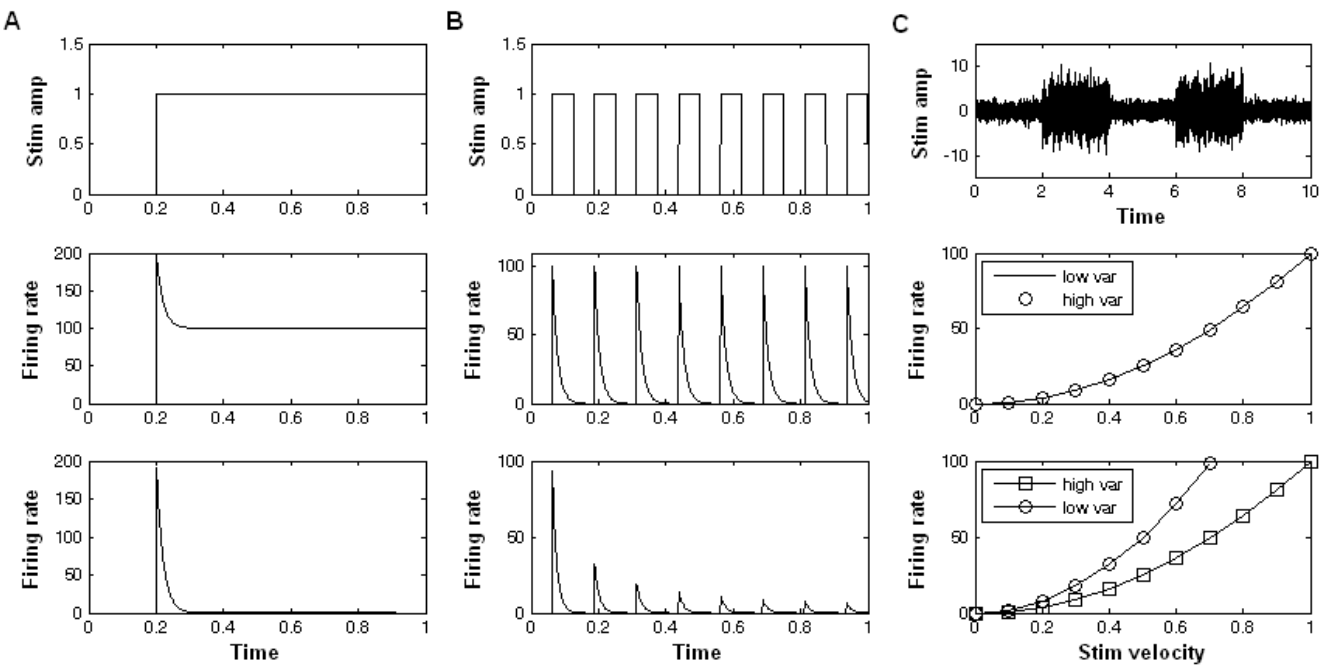

Figure 5. Adaptation in the whisker system. A. Adaptation to constant stimulus (top). Slow adapting (SA) neurons continue to respond as long as the stimulus lasts (middle), whereas rapidly adapting (RA) neurons respond only to its onset (bottom). B. Adaptation to a repetitive stimulus (top). A non-adapting ganglion-type response (middle), compared to an adapting cortex-type response (bottom). C. Adaptation to a white noise stimulus consisting of alternating low and high variance epochs (top). The tuning curve to velocity is measured separately for each epoch type. The tuning curve of a non-adapting neuron (middle) is the same under high and low variance conditions. That of an adapting neuron (bottom) rescales to the variance. All units arbitrary. 